\title{
Stability of switched linear differential systems
}

\author{
J.C. Mayo-Maldonado, P. Rapisarda and P. Rocha
}

\begin{abstract}
We study the stability of switched systems whose dynamic modes are described by systems of higherorder linear differential equations not necessarily sharing the same state space. Concatenability of trajectories at the switching instants is specified by gluing conditions, i.e. algebraic conditions on the trajectories and their derivatives at the switching instants. We provide sufficient conditions for stability based on LMIs for systems with general gluing conditions. We also analyse the role of positive-realness in providing sufficient polynomial-algebraic conditions for stability of two-modes switched systems with special gluing conditions.
\end{abstract}

Index Terms-Switched systems; behaviours; LMIs; quadratic differential forms; positive-realness.

\section{INTRODUCTION}

In established approaches, switched systems consist of a bank of state-space or descriptor form representations (see [8], [11], [28], [29]) sharing a common global state space, together with a supervisory system determining which of the modes is active. In many situations, modelling switched systems with state representations sharing a common state is justified from first principles. For example, when dealing with switched electrical circuits, it can be necessary to consider the state of the overall circuit in order to model the transitions between the different dynamical regimes. However, in other situations modelling a switched system using a common global state space is not justified by physical considerations. For example, in a multi-controller control system consisting of a plant and a bank of controllers which have different orders, the dynamical regimes have different state space dimensions. Such a system can be modelled using a global state space common to the different dynamics and reset maps (see [8]); however, there is no compelling reason to use such augmented representations, since at any given time only one controller is active. In hybrid

J.C. Mayo-Maldonado and P. Rapisarda are with the CSPC group, School of Electronics and Computer Science, University of Southampton, Great Britain, e-mail: jcmm1g11, pr3eecs.soton.ac.uk, Tel: +(44)2380593367, Fax: +(44)2380594498.

P. Rocha is with the Department of Electrical and Computer Engineering, Faculty of Engineering, University of Oporto, Portugal, e-mail: mprocha@fe.up.pt, Tel: +(351)225081844, Fax: $+(351) 225081443$. renewable energy conversion systems (see e.g. [32]) several energy sources are connected to power devices in order to transform and deliver energy to a grid. Due to the intermittent nature of renewable energies, the need arises to connect or disconnect dynamical conversion systems such as wind turbines, photovoltaic/fuel cells, etc., whose mathematical models have different orders. A similar situation arises in distributed power systems [37], where different electrical loads are connected or disconnected from a power source. Modelling such systems using a global state variable results in a more complex (more variables and more equations) dynamical model than alternative representations. For instance, such a description of a distributed power system would include the state variables of each possible load, even though in general not all loads are connected at the same time. This approach also scores low on modularity, i.e. the independent development and incremental combination of models.

Another issue with the classical approach to switched systems is that modelling from first principles usually does not yield a state-space description (for a detailed elaboration of this position see [35]). A system is the interconnection of subsystems; to model it one first describes the subsystems and the interconnection laws, possibly hierarchically repeating such procedure until simple representations (e.g. derived from a library or from elementary physical principles) can be used. Such a model typically involves algebraic relations (e.g. kinematic or equilibrium constraints), and differential equations of first- and second-order (e.g., constitutive equations of electrical components, dynamics of masses), or of higher-order (e.g., resulting from the elimination of auxiliary variables).

These considerations motivate the development of a framework to model and analyse switched systems using higher-order models describing dynamics with different complexity. In our approach, each dynamic mode is associated with a mode behaviour, the set of trajectories that satisfy the dynamical laws of that mode. The different modes share the same variables of interest ("external variables") w. A switching signal determines when a transition between dynamic modes occurs. To be admissible for the switched behaviour, a trajectory must satisfy two conditions. Firstly, it must satisfy the laws of 
the mode active in the interval between two consecutive switching instants. Secondly, at the switching instants the trajectory must satisfy certain gluing conditions, representing the physical constraints imposed by the switch, e.g. conservation of charge, kinematic constraints, and so forth. The set of all admissible trajectories is the switched behaviour, and is the central object of study in our framework.

Following the preliminary investigations for systems with one variable reported in [21], [23], in this paper we propose such a framework for the linear multivariable autonomous case. Each mode behaviour is represented by a set of linear, constant-coefficient higher-order differential equations. The gluing conditions consist of algebraic equations involving the values of a trajectory and its derivatives before and after the switching instant. We focus on closed systems, i.e. systems without input variables, and we study their Lyapunov stability using quadratic functionals of the system variables and their derivatives. We present new sufficient conditions based on systems of LMIs for the existence of a higherorder quadratic Lyapunov function for arbitrary gluing conditions. Such systems of LMIs can be set up straightforwardly from the equations of the modes and the gluing conditions. We also study the relation of positive-realness with the stability of a class of ("standard") two-modes switched systems; these conditions are multivariable generalisations of those presented in the scalar case in [21], [23]. Finally, we introduce the notion of positivereal completion of a given transfer function.

Following the behavioural approach for linear systems (see [19]), the mode equations and the gluing conditions are represented by one-variable polynomial matrices, and the Lyapunov functionals by two-variable ones. The calculus of such functionals and representations is a powerful tool conducive to the use of computer algebra techniques for the modelling and analysis of switched systems.

The approaches closest to ours are those of Geerts and Schumacher (see [6], [7]) on impulsive-smooth systems and polynomial representations; and that of Trenn about linear differential-algebraic equations (DAE's; see [12], [28], [29], [30]), and most pertinently his recent publication [31] (also worth mentioning is [2], which however is less related to our setting). These authors consider mode dynamics with different state-space dimension, a situation generally involving impulses in the system trajectories, a relevant issue also for practical reasons (see e.g. [5]). In [8], [9], [38], switched systems with impulsive effects are considered to be those that allow discontinuities ("state jumps") in the state trajectories. On the other hand, in [28], [34], Dirac impulses (or their derivatives) are explicitly considered in the solutions of the system variables. In [28] a unifying, rigorous distributional framework for switched systems has been given, this approach encompasses also the detection of impulses directly from the equations. Similarly, for higher-order representations as in [31], the jumps and impulses induced by the system equations together with additional impact maps are used to specify the impulsive part of the behaviour. Stability (also in a Lyapunov sense) for impulse-free switched DAE's has been presented in [12].

In this paper we deal with autonomous (i.e. closed) modes; impulsive effects are implicitly defined by the gluing conditions and the mode dynamics involved in the transition (i.e. do not depend for example on the degree of differentiability of some input variable). Our position is that gluing conditions are a given; we take them at face value. Whether they are physically meaningful or not; whether they imply impulses or not; and whether the latter is an important issue for the particular physical system at hand, are major modelling issues that we assume have been weighed carefully by the modeller (on this issue see also p. 749 of [6]). In certain cases, see Examples 1 and 2 below, our attitude towards gluing conditions seems to involve less conceptual difficulties than letting the equations to dictate the re-initialisation mechanism at the switching instants. This "agnostic" position does not absolve us though from the important task, relevant for instance in the case of models assembled from libraries, of studying how to determine the presence of impulses directly from the equations and associated gluing conditions; this is a pressing research question to be considered elsewhere (on this issue see [28], [29]).

We study stability for higher-order representations also in the presence of impulsive effects in the sense of [8], [9], [38], i.e. allowing discontinuities in the trajectories. However, we do recognise the validity of the position taken in [29], that in a system with Dirac impulses small initial states produce unbounded state trajectories, thus leading to instability. Note also that other recent approaches are focused on switched systems whose trajectories are everywhere continuous, and thus not contain neither jumps nor impulses; e.g. [40], where a complete framework for dissipative switched systems is presented (see Sec. II ibid.).

The paper is organised as follows: in section II we define switched linear differential systems (SLDS), we give examples of SLDS, and we discuss the issue of wellposedness. In section III we give sufficient conditions for stability of a SLDS based on the existence of a multiple Lyapunov function (MLF). We also discuss how to com- 
pute MLFs using LMIs. In section IV we focus on twomodes SLDS, and we investigate the role of positiverealness in establishing the stability of such systems. The notational conventions and some background material on the behavioural approach and quadratic differential forms are gathered in Appendix I, while the proofs are gathered in Appendix II.

\section{Switched Autonomous Linear DifFERENTIAL SYSTEMS}

\section{A. Basic definitions}

Recall from App. A-B the definition of $\mathfrak{L}^{\mathrm{w}}$ as the set of linear differential behaviours. A switched linear differential system is defined in the following way (see also [21], [23]).

Definition 1. A switched linear differential system $(S L D S) \Sigma$ is a quadruple $\Sigma=\{\mathcal{P}, \mathcal{F}, \mathcal{S}, \mathcal{G}\}$ where $\mathcal{P}=\{1, \ldots, N\} \subset \mathbb{N}$ is the set of indices; $\mathcal{F}=$ $\left\{\mathfrak{B}_{1}, \ldots, \mathfrak{B}_{N}\right\}$, with $\mathfrak{B}_{j} \in \mathfrak{L}^{\text {w }}$ for $j \in \mathcal{P}$ is the bank of behaviours; $\mathcal{S}=\{s: \mathbb{R} \rightarrow \mathcal{P} \mid$ $s$ is piecewise constant and right-continuous $\}$, is the set of admissible switching signals; and

$$
\begin{gathered}
\mathcal{G}=\left\{\left(G_{k \rightarrow \ell}^{-}(\xi), G_{k \rightarrow \ell}^{+}(\xi)\right) \in \mathbb{R}^{\bullet \times \mathrm{w}}[\xi] \times \mathbb{R}^{\bullet \times \mathrm{w}}[\xi]\right. \\
\mid 1 \leq k, \ell \leq N, k \neq \ell\},
\end{gathered}
$$

is the set of gluing conditions. The set of switching instants associated with $s \in \mathcal{S}$ is defined by $\mathbb{T}_{s}:=$ $\left\{t \in \mathbb{R} \mid \lim _{\tau}{ }_{t} s(\tau) \neq s(t)\right\}=\left\{t_{1}, t_{2}, \ldots\right\}$, where $t_{i}<t_{i+1}$.

A SLDS induces a switched behaviour, defined as follows.

Definition 2. Let $\Sigma=\{\mathcal{P}, \mathcal{F}, \mathcal{S}, \mathcal{G}\}$ be a SLDS, and let $s \in \mathcal{S}$. The $s$-switched linear differential behaviour $\mathfrak{B}^{s}$ is the set of trajectories $w: \mathbb{R} \rightarrow \mathbb{R}^{\mathrm{w}}$ that satisfy the following two conditions:

1) for all $t_{i}, t_{i+1} \in \mathbb{T}_{s},\left.\left.w\right|_{\left[t_{i}, t_{i+1}\right)} \in \mathfrak{B}_{s\left(t_{i}\right)}\right|_{\left[t_{i}, t_{i+1}\right)}$;

2) $w$ satisfies the gluing conditions $\mathcal{G}$ at the switching instants for each $t_{i} \in \mathbb{T}_{s}$, i.e. $\left(G_{s\left(t_{i-1}\right) \rightarrow s\left(t_{i}\right)}^{+}\left(\frac{d}{d t}\right)\right) w\left(t_{i}^{+}\right)=$ $\left(G_{s\left(t_{i-1}\right) \rightarrow s\left(t_{i}\right)}^{-}\left(\frac{d}{d t}\right)\right) w\left(t_{i}^{-}\right)$.

The switched linear differential behaviour $(S L D B) \mathfrak{B}^{\Sigma}$ of $\Sigma$ is defined by $\mathfrak{B}^{\Sigma}:=\bigcup_{s \in \mathcal{S}} \mathfrak{B}^{s}$.

We make the standard assumption (see e.g. sect. 1.3.3 of [26]) that the number of switching instants in any finite interval of $\mathbb{R}$ is finite. Moreover, in this paper we assume that the behaviours $\mathfrak{B}_{i}$, for all $i \in \mathcal{P}$ are autonomous. Since the trajectories of an autonomous behaviour are infinitely differentiable (see 3.2.16 of [19]), the trajectories of a switched behaviour as in Def.
2 are smooth in any interval between two consecutive switching times.

We now give three examples of switched behaviours; besides exemplifying the Definitions, they allow us to point out some important features of our approach to switched systems (see also section III for another more realistic example).

Example 1. Consider the electrical circuit in Fig. 1, where $C=1 F, R=\frac{1}{2} \Omega$ and $w_{1}$ and $w_{2}$ are voltages.

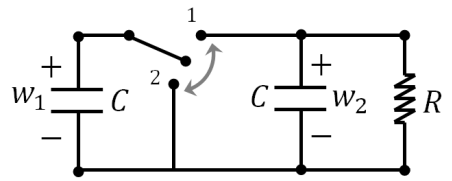

Fig. 1. An electrical circuit

With the switch in position 1 , the dynamical equations are

$$
\begin{aligned}
\frac{d}{d t} w_{2}+w_{2} & =0 \\
w_{1}-w_{2} & =0 ;
\end{aligned}
$$

when the switch is in position 2, the dynamical equations are

$$
\begin{aligned}
\frac{d}{d t} w_{2}+w_{2} & =0 \\
w_{1} & =0 .
\end{aligned}
$$

If we consider the voltage across the capacitors as the variables of interest, we then define $\mathfrak{B}_{1}:=$ ker $\left[\begin{array}{cc}0 & \frac{d}{d t}+1 \\ 1 & -1\end{array}\right]$ and $\mathfrak{B}_{2}:=\operatorname{ker}\left[\begin{array}{cc}0 & \frac{d}{d t}+1 \\ 1 & 0\end{array}\right]$. The switched behaviour consists of all piecewise smooth functions $\operatorname{col}\left(w_{1}, w_{2}\right)$ that satisfy (1) or (2) depending on the position of the switch, and that at the switching instant satisfy the gluing conditions that follow from the principle of conservation of charge (see [4]), i.e. either $w_{1}\left(0^{+}\right)=\frac{1}{2} w_{2}\left(0^{-}\right), w_{2}\left(0^{+}\right)=\frac{1}{2} w_{2}\left(0^{-}\right)$(for a transition $\left.\mathfrak{B}_{2} \rightarrow \mathfrak{B}_{1}\right)$ or $w_{1}\left(0^{+}\right)=0, w_{2}\left(0^{+}\right)=w_{2}\left(0^{-}\right)$ (for a transition $\mathfrak{B}_{1} \rightarrow \mathfrak{B}_{2}$ ). The corresponding matrices are

$$
G_{2 \rightarrow 1}^{-}:=\left[\begin{array}{cc}
0 & \frac{1}{2} \\
0 & \frac{1}{2}
\end{array}\right], G_{2 \rightarrow 1}^{+}:=I_{2},
$$

and

$$
G_{1 \rightarrow 2}^{-}:=\left[\begin{array}{ll}
0 & 0 \\
0 & 1
\end{array}\right], G_{1 \rightarrow 2}^{+}:=I_{2} .
$$

These gluing conditions imply that in any non-trivial case the value of $w_{1}$ jumps at the switching instant. 
Example 2. Consider two behaviours respectively described by the equations

$$
\begin{aligned}
\frac{d}{d t} w_{2}+w_{2} & =0 \\
w_{1}-w_{2} & =0,
\end{aligned}
$$

and

$$
\begin{aligned}
\frac{d}{d t} w_{1}+\frac{d}{d t} w_{2}+w_{1}+w_{2} & =0 \\
w_{1} & =0
\end{aligned}
$$

The gluing conditions for a transition $\mathfrak{B}_{2} \rightarrow \mathfrak{B}_{1}$ are associated with the matrices

$$
G_{2 \rightarrow 1}^{-}:=\left[\begin{array}{ll}
0 & 1 \\
0 & 1
\end{array}\right], G_{2 \rightarrow 1}^{+}:=I_{2},
$$

and for a transition $\mathfrak{B}_{1} \rightarrow \mathfrak{B}_{2}$ they are defined by

$$
G_{1 \rightarrow 2}^{-}:=\left[\begin{array}{cc}
0 & 0 \\
\frac{1}{2} & \frac{1}{2}
\end{array}\right], G_{1 \rightarrow 2}^{+}:=I_{2} ;
$$

i.e. in a switch $\mathfrak{B}_{1} \rightarrow \mathfrak{B}_{2}$ the new value of $w_{2}$ is the average of the old values of $w_{1}$ and $w_{2}$.

Examples 1 and 2 offer the opportunity of making two important remarks.

Remark 1. Note that (2) and (6) describe the same set of solutions; indeed, the description (2) can be obtained from (6) by unimodular operations, which in the case of autonomous systems do not alter the solution set (see Th. 2.5.4 and Th. 3.2.16 of [19]) ${ }^{1}$. Considering that (1) and (5) are equivalent, the dynamic modes are the same for both switched systems; thus the two switched behaviours are different because the gluing conditions are. We will prove later in this paper that these two switched systems also have different stability propertiesthat of Ex. 1 is stable under arbitrary switching signals, while the other is not. Stability arises from the interplay of mode dynamics and gluing conditions.

Remark 2. Gluing conditions should be defined on the basis of the physics of the system under study. Those for the system of Example 1 are meaningful for the particular physical system at hand. However, for another physical system whose modes happen to be described also by (5)-(6), the conditions (7)-(8) may also be physically plausible. In each case we assume that wellgrounded physical considerations have been motivating the choice.

\footnotetext{
${ }^{1}$ Some equivalence results for the $\mathfrak{C}^{\infty}$-case are not valid for nonautonomous systems and $\mathcal{L}_{1}^{\text {loc }}$ trajectories; see for example [18]. On equivalence of polynomial representations of switched systems, see sect. 3 of [7].
}

\section{B. Well-posedness of gluing conditions}

In principle Def.s 1 and 2 do not restrict the gluing conditions; however, since we assume that the modes are autonomous, i.e. no external influences are applied to the system between consecutive switching times, it is reasonable to require more. Namely, no different admissible trajectories should exist with the same past (i.e. same mode transitions at the same switching instants, and same restrictions from $t=-\infty$ up until a given switching instant $\bar{t}$ ). If such trajectories exist, then at $\bar{t}$ the past "splits" in different futures; however, since no external inputs could trigger such a change, the past of a trajectory should uniquely define its future. These considerations lead to the concept of well-posed gluing conditions, which we now introduce.

In order to do so, we first choose kernel representations $\mathfrak{B}_{k}=$ : $\operatorname{ker} R_{k}\left(\frac{d}{d t}\right)$, with $R_{k} \in \mathbb{R}^{\mathrm{w} \times \mathrm{w}}[\xi]$ nonsingular, $k=1, \ldots, N$ for the modes. We also define $n_{k}:=$ $\operatorname{deg}\left(\operatorname{det}\left(R_{k}\right)\right), k=1, \ldots, N$, and we choose minimal state maps (see App. A-C) $X_{k} \in \mathbb{R}^{n_{k} \times \mathrm{w}}[\xi], k=1, \ldots, N$. Every polynomial differential operator $G\left(\frac{d}{d t}\right)$ on $\mathfrak{B}_{k}$ has a unique $R_{k}$-canonical representative $G^{\prime}\left(\frac{d}{d t}\right)$, denoted by $G^{\prime}=G \bmod R_{k}$, such that $G^{\prime}\left(\frac{d}{d t}\right) w=G\left(\frac{d}{d t}\right) w$ for all $w \in \mathfrak{B}_{k}$ (see App. A-B). Now let $\left(G_{k \rightarrow \ell}^{-}, G_{k \rightarrow \ell}^{+}\right) \in \mathcal{G}$; then $\left(G_{k \rightarrow \ell}^{-} \bmod R_{k}, G_{k \rightarrow \ell}^{+} \bmod R_{\ell}\right)$ are equivalent to $\left(G_{k \rightarrow \ell}^{-}, G_{k \rightarrow \ell}^{+}\right)$, in the sense that the algebraic conditions imposed by the one pair are satisfied iff they are satisfied by the other. Moreover, since $G_{k \rightarrow \ell}^{-} \bmod R_{k}$ and $G_{k \rightarrow \ell}^{+} \bmod R_{\ell}$ are $R_{k^{-}}$, respectively $R_{\ell}$-canonical, there exist constant matrices $F_{k \rightarrow \ell}^{-}$and $F_{k \rightarrow \ell}^{+}$of suitable dimensions such that $G_{k \rightarrow \ell}^{-}(\xi) \bmod R_{k}=F_{k \rightarrow \ell}^{-} X_{k}(\xi)$ and $G_{k \rightarrow \ell}^{+}(\xi) \bmod R_{\ell}=F_{k \rightarrow \ell}^{+} X_{\ell}(\xi)$. We call

$\mathcal{G}^{\prime}:=\left\{\left(F_{k \rightarrow \ell}^{-} X_{k}(\xi), F_{k \rightarrow \ell}^{+} X_{\ell}(\xi)\right) \mid 1 \leq k, \ell \leq N, k \neq \ell\right\}$

the normal form of $\mathcal{G}$.

Definition 3. Let $\Sigma$ be a SLDS with $\mathfrak{B}_{i}=\operatorname{ker} R_{i}\left(\frac{d}{d t}\right)$ autonomous, $i=1, \ldots, N$. The normal form gluing conditions $\mathcal{G}^{\prime}:=\left\{\left(F_{k \rightarrow \ell}^{-} X_{k}(\xi), F_{k \rightarrow \ell}^{+} X_{\ell}(\xi)\right)\right\}_{k, \ell=1, \ldots, N, k \neq \ell}$ are well-posed if for all $k, \ell=1, \ldots, N, k \neq \ell$, and for all $v_{k} \in \mathbb{R}^{n_{k}}$ there exists at most one $v_{\ell} \in \mathbb{R}^{n_{\ell}}$ such that $F_{k \rightarrow \ell}^{-} v_{k}=F_{k \rightarrow \ell}^{+} v_{\ell}$.

Thus if a transition occurs between $\mathfrak{B}_{k}$ and $\mathfrak{B}_{\ell}$ at $t_{j}$, and if an admissible trajectory ends at a "final state" $v_{k}=X_{k}\left(\frac{d}{d t}\right) w\left(t_{j}^{-}\right)$, then there exists at most one "initial state" for $\mathfrak{B}_{\ell}$, defined by $X_{\ell}\left(\frac{d}{d t}\right) w\left(t_{j}^{+}\right):=v_{\ell}$, compatible with the gluing conditions.

Example 3. Depending on the value of a switching signal a plant $\Sigma_{P}$ with two external variables, described by the differential equation $\frac{d}{d t} w_{1}-w_{1}-w_{2}=0$, is connected with one of two possible controllers $\Sigma_{C_{1}}$ and 
$\Sigma_{C_{2}}$, described respectively by $-3 \frac{d}{d t} w_{1}-w_{1}-\frac{d}{d t} w_{2}=0$ and $-2 w_{1}-w_{2}=0$. Depending on which controller is active, the resulting closed-loop behaviours are

$$
\mathfrak{B}_{1}:=\operatorname{ker}\left[\begin{array}{cc}
\frac{d}{d t}-1 & -1 \\
-3 \frac{d}{d t}-1 & -\frac{d}{d t}
\end{array}\right],
$$

and

$$
\mathfrak{B}_{2}:=\operatorname{ker}\left[\begin{array}{cc}
\frac{d}{d t}-1 & -1 \\
-2 & -1
\end{array}\right] \text {. }
$$

Note that $\mathfrak{B}_{1}$ and $\mathfrak{B}_{2}$ have different McMillan degrees (2 and 1, respectively). We define the gluing conditions for the SLDS associated with $\mathfrak{B}_{1}$ and $\mathfrak{B}_{2}$ by

$$
\begin{gathered}
G_{2 \rightarrow 1}^{-}(\xi):=\left[\begin{array}{cc}
0 & 1 \\
0 & -2
\end{array}\right], G_{2 \rightarrow 1}^{+}(\xi):=\left[\begin{array}{ll}
0 & 1 \\
1 & 0
\end{array}\right], \\
G_{1 \rightarrow 2}^{-}(\xi):=\left[\begin{array}{ll}
1 & 0
\end{array}\right], G_{1 \rightarrow 2}^{+}(\xi):=\left[\begin{array}{ll}
1 & 0
\end{array}\right] .
\end{gathered}
$$

The rationale underlying our choice of gluing conditions is that any trajectory of $\mathfrak{B}_{1}$ is uniquely specified by the instantaneous values of $\operatorname{col}\left(w_{1}, w_{2}\right)$, while a trajectory of $\mathfrak{B}_{2}$ is uniquely specified by the instantaneous value of $w_{1}$. Moreover, when switching from the dynamics of $\mathfrak{B}_{1}$ to those of $\mathfrak{B}_{2}$, we require that the values of $w_{1}$ before and after the switching instant coincide. In a switch from $\mathfrak{B}_{2}$ to $\mathfrak{B}_{1}$, since the second differential equation describing $\mathfrak{B}_{2}$ yields $w_{2}=-2 w_{1}$ before the switch, we impose that $w_{2}\left(t_{k}^{+}\right)=w_{2}\left(t_{k}^{-}\right)=-2 w_{1}\left(t_{k}^{-}\right)$. Moreover, note that a minimal state map for $\mathfrak{B}_{1}$ is $X_{1}(\xi):=I_{2}$, and a minimal state map for $\mathfrak{B}_{2}$ is $X_{2}(\xi)=\left[\begin{array}{ll}1 & 0\end{array}\right]$; and $G_{2 \rightarrow 1}^{+} \bmod R_{1}(\xi)=G_{2 \rightarrow 1}^{+}(\xi)=F_{2 \rightarrow 1}^{+} X_{1}(\xi):=$ $\left[\begin{array}{ll}0 & 1 \\ 1 & 0\end{array}\right]_{2}^{I_{2}}$. Similarly, $G_{1 \rightarrow 2}^{+} \bmod R_{2}(\xi)=G_{1 \rightarrow 2}^{+}(\xi)=$ $F_{1 \rightarrow 2}^{+} X_{2}(\xi):=1\left[\begin{array}{ll}1 & 0\end{array}\right]$. Consequently, these gluing conditions are well-posed. It can be verified in a similar way that the gluing conditions of Examples 1 and 2 are also well-posed.

Remark 3. The definition of well-posedness concerns uniqueness of an admissible "initial condition" $v_{\ell}$ in $\mathfrak{B}_{\ell}$ for a given "final condition" $v_{k}$ in $\mathfrak{B}_{k}$. However, another important issue is existence of such admissible initial condition at the switching instant. Note for instance that it may happen that the gluing conditions cannot be satisfied by nonzero trajectories; they may not be "consistent" with the mode dynamics. As an example of such situation, consider a SLDS with modes (5) and (6), and (well-posed) gluing conditions $G_{2 \rightarrow 1}^{-}:=$ $I_{2}, G_{2 \rightarrow 1}^{+}:=I_{2}, G_{1 \rightarrow 2}^{-}:=I_{2}, G_{1 \rightarrow 2}^{+}:=I_{2} . w \in \mathfrak{B}_{1}$ iff $w(t)=\alpha \operatorname{col}\left(e^{-t}, e^{-t}\right), \alpha \in \mathbb{R}$; and $w \in \mathfrak{B}_{2}$ iff $w(t)=\alpha \operatorname{col}\left(e^{-t}, 0\right), \alpha \in \mathbb{R}$. Since constant switching signals $\sigma_{1}=1$ and $\sigma_{2}=2$ are admissible, it follows that $\mathfrak{B}^{\Sigma} \supset \mathfrak{B}_{i}, i=1,2$. However, no genuine switched trajectory exists besides the zero one, since the gluing conditions cannot be satisfied by nonzero trajectories of either of the behaviours. The problem whether a given "initial condition" is consistent or not with the mode dynamics was solved most satisfactorily in the switched DAE's framework of Trenn (see Ch. 4 of [28]); algorithms are stated that from the matrices describing a mode compute "consistency projectors" whose image is the subspace of consistent initial values. We briefly discuss the issue of consistent gluing conditions in our framework.

Denote the roots of det $R_{k}(\xi)$ by $\lambda_{k, i}, i=1, \ldots n_{k}$. We assume for ease of exposition that the algebraic multiplicity of $\lambda_{k, i}$ equals the dimension of ker $R_{k}\left(\lambda_{k, i}\right)$. It follows from sect. 3.2.2 of [19] that $w \in \mathfrak{B}_{k}$ iff there exist $\alpha_{k, i} \in \mathbb{C}, i=1, \ldots, n_{i}$ such that $w=$ $\sum_{i=1}^{n_{k}} \alpha_{k, i} w_{k, i} \exp _{\lambda_{i} t}$, where $w_{k, i} \in \mathbb{C}^{\mathrm{w}}$ is such that $R_{k}\left(\lambda_{k, i}\right) w_{k, i}=0$, and the $w_{k, i}$ associated with equal $\lambda_{k, i}$ are linearly independent. Note that the $\alpha_{k, i}$ associated to conjugate $\lambda_{k, i}$ are conjugate.

Define $V_{i}:=\left[\begin{array}{lll}X_{i}\left(\lambda_{i, 1}\right) w_{i, 1} & \ldots & X_{i}\left(\lambda_{i, n_{i}}\right) w_{i, n_{i}}\end{array}\right] \in$ $\mathbb{C}^{n_{i} \times n_{i}}$ and $\alpha_{i}:=\left[\begin{array}{lll}\alpha_{i, 1} & \ldots & \alpha_{i, n_{i}}\end{array}\right]^{\top}, i=k, \ell ;$ and consider a switch from $\mathfrak{B}_{k}$ to $\mathfrak{B}_{\ell}$ at $t=0$. The gluing conditions require that $G_{k \rightarrow \ell}^{-}(w)\left(0^{-}\right)=F_{k \rightarrow \ell}^{-} V_{k} \alpha_{k}=$ $F_{k \rightarrow \ell}^{+} V_{\ell} \alpha_{\ell}=G_{k \rightarrow \ell}^{+}(w)\left(0^{+}\right)$. Such $\alpha_{i}, i=k, \ell$ exist if and only if im $F_{k \rightarrow \ell}^{-} V_{k} \subseteq \operatorname{im~} F_{k \rightarrow \ell}^{+} V_{\ell}$. Standard arguments in ordinary differential equations show that $V_{k}$ and $V_{\ell}$ are nonsingular; consequently the consistency condition can be equivalently stated as im $F_{k \rightarrow \ell}^{-} \subseteq$ im $F_{k \rightarrow \ell}^{+}$.

Well-posedness implies that for all $k, \ell=1, \ldots, N$, $k \neq \ell, F_{k \rightarrow \ell}^{+}$is full column rank, and consequently there exists a re-initialisation map $L_{k \rightarrow \ell}: \mathbb{R}^{n_{k}} \rightarrow \mathbb{R}^{n_{\ell}}$ defined by $L_{k \rightarrow \ell}:=F_{k \rightarrow \ell}^{+*} F_{k \rightarrow \ell}^{-}$, where $F_{k \rightarrow \ell}^{+*}$ is a left inverse of $F_{k \rightarrow \ell}^{+}$. For all $t_{j} \in \mathbb{T}_{s}$ and all admissible $w \in \mathfrak{B}^{\Sigma}$ it holds that

$$
\begin{gathered}
{\left[s\left(t_{j-1}\right)=k, s\left(t_{j}\right)=\ell\right] \text { and }} \\
{\left[G_{k \rightarrow \ell}^{+}\left(\frac{d}{d t}\right) w\left(t_{j}^{+}\right)=G_{k \rightarrow \ell}^{-}\left(\frac{d}{d t}\right) w\left(t_{j}^{-}\right)\right]} \\
\Longrightarrow\left[X_{\ell}\left(\frac{d}{d t}\right) w\left(t_{j}^{+}\right)=L_{k \rightarrow \ell}\left(X_{k}\left(\frac{d}{d t}\right) w\left(t_{j}^{-}\right)\right)\right] .
\end{gathered}
$$

Note that the re-initialisation map is not uniquely determined unless $F_{k \rightarrow \ell}^{+}$is nonsingular. In the rest of the paper, we assume well-posed gluing conditions with fixed re-initialisation maps.

\section{MUlTiPle LyAPUNOV FUNCTIONS FOR SLDS}

We call a SLDB $\mathfrak{B}^{\Sigma}$ (and by extension, the SLDS $\Sigma)$ asymptotically stable if $\lim _{t \rightarrow+\infty} w(t)=0$ for all $w \in \mathfrak{B}^{\Sigma}$. It follows from this definition and the fact that arbitrary switching signals are considered, that in 
an asymptotically stable SLDS, all mode behaviours $\mathfrak{B}_{i}$ must be asymptotically stable and consequently autonomous (see [19], sec. 7.2). Asymptotic stability for linear differential behaviours can be proved by producing a higher-order quadratic Lyapunov function, i.e. a quadratic differential function (QDF) $Q_{\Psi}$ such that $Q_{\Psi} \stackrel{\mathfrak{B}}{\geq} 0$ and $\frac{d}{d t} Q_{\Psi} \stackrel{\mathfrak{B}}{<} 0$, see sect. 4 of [36]. The next result gives a sufficient condition for stability of SLDS in terms of quadratic multiple Lyapunov functions (MLFs) (see e.g [13] and sect. III.B of [14]).

Theorem 1. Let $\Sigma$ be a SLDS (see Def. 1). Assume that there exist $Q D F s Q_{\Psi_{i}}, i=1, \ldots, N$ such that

1. $Q_{\Psi_{i}} \stackrel{\mathfrak{B}_{i}}{\geq} 0, i=1, \ldots, N$;

2. $\frac{d}{d t} Q_{\Psi_{i}} \stackrel{\mathfrak{B}_{i}}{<} 0, i=1, \ldots, N$;

3. $\forall w \in \mathfrak{B}^{\Sigma}$ and $\forall t_{j} \in \mathbb{T}_{s}, Q_{\Psi_{s\left(t_{j-1}\right)}}(w)\left(t_{j}^{-}\right) \geq$ $Q_{\Psi_{s\left(t_{j}\right)}}(w)\left(t_{j}^{+}\right)$.

Then $\Sigma$ is asymptotically stable.

Proof: See Appendix B.

Conditions 1 and 2 of Th. 1 are equivalent to $Q_{\Psi_{i}}$ being a Lyapunov function for $\mathfrak{B}_{i}, i=1, \ldots, N$. Condition 3 requires that the value of the multiple functional associated to $Q_{\Psi_{i}}, i=1, \ldots, N$, does not increase at the switching instants.

We now describe a procedure, based on the calculus of QDFs and on LMIs, to compute an MLF as in Th. 1. We first recall the following result from [36], that reduces the computation of quadratic Lyapunov functions to the solution of two-variable polynomial equations.

Theorem 2. Let $\mathfrak{B}=\operatorname{ker} R\left(\frac{d}{d t}\right)$, with $R \in \mathbb{R}^{\mathrm{w} \times \mathrm{w}}[\xi]$ nonsingular. If $\mathfrak{B}$ is asymptotically stable, for every $Q \in$ $\mathbb{R}^{\bullet \times \mathrm{w}}[\xi]$ there exist $\Psi \in \mathbb{R}_{s}^{\mathrm{w} \times \mathrm{w}}[\zeta, \eta]$ and $Y \in \mathbb{R}^{\mathrm{w} \times \mathrm{w}}[\xi]$ such that $Q_{\Psi} \geq 0$ and

$(\zeta+\eta) \Psi(\zeta, \eta)=Y(\zeta)^{\top} R(\eta)+R(\zeta)^{\top} Y(\eta)-Q(\zeta)^{\top} Q(\eta)$

If either one of $Q$ or $Y$ is $R$-canonical, then also the other and $\Psi$ are $R$-canonical. Moreover if rank $\operatorname{col}(R(\lambda), Q(\lambda))=$ w for all $\lambda \in \mathbb{C}$ such that $\operatorname{det} R(\lambda)=0$, then $Q_{\Psi} \stackrel{\mathfrak{B}}{>} 0$.

Proof: The result follows from Th. 4.8 and Th. 4.12 of [36].

Thus, a quadratic Lyapunov function $Q_{\Psi}$ can be computed by choosing some $Q$ and solving the polynomial Lyapunov equation (PLE) (9). Algebraic methods for solving it are illustrated in [16]; we devise an LMI-based one more suitable to our purposes. We first introduce the following important lemma.
Lemma 1. Let $\mathfrak{B}=\operatorname{ker} R\left(\frac{d}{d t}\right)$, with $R \in \mathbb{R}^{\mathrm{w} \times \mathrm{w}}[\xi]$ nonsingular. Let $X \in \mathbb{R}^{n \times \mathrm{w}}[\xi]$ be a minimal state map for $\mathfrak{B}$. Assume that $\mathfrak{B}$ is asymptotically stable. Let $Q, Y$, and $\Psi$ satisfy (9), and assume that either $Q$ or $Y$ is $R$ canonical. There exist $\bar{K}=\bar{K}^{\top} \in \mathbb{R}^{n \times n}, \bar{Y} \in \mathbb{R}^{\mathrm{w} \times n}$, $\bar{Q} \in \mathbb{R}^{\bullet \times n}$ such that $\Psi(\zeta, \eta)=X(\zeta)^{\top} \bar{K} X(\eta), Y(\xi)=$ $\bar{Y} X(\xi)$, and $Q(\xi)=\bar{Q} X(\xi)$.

Proof. See Appendix B.

Now we proceed to relate the two-variable polynomial equation (9), with a constant matrix equation.

Proposition 1. Under the assumptions of Lemma 1, write $\Psi(\zeta, \eta)=X(\zeta)^{\top} \bar{K} X(\eta), Y(\xi)=\bar{Y} X(\xi)$, and $Q(\xi)=\bar{Q} X(\xi)$, and $R(\xi)=\sum_{i=0}^{L} R_{i} \xi^{i}$, with $R_{i} \in$ $\mathbb{R}^{\mathrm{w} \times \mathrm{w}}, i=0, \ldots, L$; then there exist $X_{i} \in \mathbb{R}^{n \times \mathrm{w}}, i=$ $0,1, \ldots, L-1$, such that $X(\xi)=\sum_{i=0}^{L-1} X_{i} \xi^{i}$. Moreover, denote the coefficient matrices of $R(\xi)$ and $X(\xi)$ by $\widetilde{R}:=\left[\begin{array}{lll}R_{0} & \ldots & R_{L}\end{array}\right]$ and $\widetilde{X}:=\left[\begin{array}{lll}X_{0} & \ldots & X_{L-1}\end{array}\right]$. The following statements are equivalent:

1. $\Psi(\zeta, \eta), Y(\xi)$ and $Q(\xi)$ satisfy (9);

2. There exist $\bar{K}=\bar{K}^{\top} \in \mathbb{R}^{n \times n}, \bar{Y} \in \mathbb{R}^{\mathrm{w} \times n}, \bar{Q} \in$ $\mathbb{R}^{\bullet \times n}$ such that

$$
\begin{gathered}
{\left[\begin{array}{c}
0_{\mathrm{w} \times n} \\
\widetilde{X}^{\top}
\end{array}\right] \bar{K}\left[\begin{array}{ll}
\widetilde{X} & 0_{n \times \mathrm{w}}
\end{array}\right]+\left[\begin{array}{c}
\widetilde{X}^{\top} \\
0_{\mathrm{w} \times n}
\end{array}\right] \bar{K}\left[\begin{array}{ll}
0_{n \times \mathrm{w}} & \widetilde{X}
\end{array}\right]} \\
-\left[\begin{array}{c}
\widetilde{X}^{\top} \\
0_{\mathrm{w} \times n}
\end{array}\right] \bar{Y}^{\top} \widetilde{R}-\widetilde{R}^{\top} \bar{Y}\left[\begin{array}{ll}
\widetilde{X} & 0_{n \times \mathrm{w}}
\end{array}\right] \\
+\left[\begin{array}{c}
\widetilde{X}^{\top} \\
0_{\mathrm{w} \times n}
\end{array}\right] \bar{Q}^{\top} \bar{Q}\left[\begin{array}{ll}
\widetilde{X} & 0_{n \times \mathrm{w}}
\end{array}\right]=0
\end{gathered}
$$

If moreover, rank $\operatorname{col}(R(\lambda), Q(\lambda))=\mathrm{w}$ for all $\lambda \in \mathbb{C}$, then 1) is equivalent with 2) and $\bar{K}>0$.

Proof: See Appendix B.

The following theorem shows how to compute MLFs as in Th. 1 for SLDS using LMIs. For ease of exposition we assume that for each root of $\operatorname{det} R_{k}(\xi)$ the algebraic multiplicity coincides with the geometric multiplicity.

Theorem 3. Let $\Sigma$ be a SLDS (see Def. 1), with $\mathfrak{B}_{k}=\operatorname{ker} R_{k}\left(\frac{d}{d t}\right)$ asymptotically stable, $k=1, \ldots, N$ and $R_{k} \in \mathbb{R}^{\mathrm{w} \times \mathrm{w}}[\xi]$ nonsingular. Let $X_{k} \in \mathbb{R}^{n \times \mathrm{w}}[\xi]$ be a minimal state map for $\mathfrak{B}_{k}$. Write $R_{k}(\xi)=$ $\sum_{i=0}^{L_{k}} R_{k, i} \xi^{i}$, and denote the coefficient matrix of $R_{k}(\xi)$ by $\widetilde{R}_{k}:=\left[\begin{array}{lll}R_{k, 0} & \ldots & R_{k, L_{k}}\end{array}\right]$ and that of $X_{k}(\xi)$ by $\widetilde{X}_{k}:=\left[\begin{array}{lll}X_{k, 0} & \ldots & X_{k, L_{k}-1}\end{array}\right]$. Denote the roots of det $R_{k}(\xi)$ by $\lambda_{k, i}, \quad i=1, \ldots n_{k}$. Assume that the algebraic multiplicity of $\lambda_{k, i}$ equals the dimension of $\operatorname{ker} R_{k}\left(\lambda_{k, i}\right)$. Let $w_{k, i} \in \mathbb{C}^{\mathrm{w}}$ be such that $R_{k}\left(\lambda_{k, i}\right) w_{k, i}=0$, with the $w_{k, i}$ associated with equal $\lambda_{k, i}$ linearly independent. Define $V_{k} \in \mathbb{C}^{n_{k} \times n_{k}}$ by $V_{k}:=$ $\left[\begin{array}{lll}X_{k}\left(\lambda_{k, 1}\right) w_{k, 1} & \ldots & X_{k}\left(\lambda_{k, n_{k}}\right) w_{k, n_{k}}\end{array}\right], \quad k=1, \ldots, N$. 
Denote by $L_{k \rightarrow \ell}, k, \ell=1 \ldots, N, k \neq \ell$, the reinitialisation maps of $\Sigma$.

There exist $\bar{K}_{k} \in \mathbb{R}^{n_{k} \times n_{k}}, \bar{Y}_{k} \in \mathbb{R}^{\mathrm{w} \times n_{k}}, k=1 \ldots, N$ such that

$$
\begin{aligned}
\widetilde{\Phi}_{k}:= & {\left[\begin{array}{c}
0_{\mathrm{w} \times n} \\
\widetilde{X}_{k}^{\top}
\end{array}\right] \bar{K}_{k}\left[\begin{array}{ll}
\widetilde{X}_{k} & 0_{n \times \mathrm{w}}
\end{array}\right] } \\
& +\left[\begin{array}{c}
\widetilde{X}_{k}^{\top} \\
0_{\mathrm{w} \times n}
\end{array}\right] \bar{K}_{k}\left[\begin{array}{ll}
0_{n \times \mathrm{w}} & \widetilde{X}_{k}
\end{array}\right]-\left[\begin{array}{c}
\widetilde{X}_{k}^{\top} \\
0_{\mathrm{w} \times n}
\end{array}\right] \bar{Y}_{k}^{\top} \widetilde{R}_{k} \\
& -\widetilde{R}_{k}^{\top} \bar{Y}_{k}\left[\begin{array}{ll}
\widetilde{X}_{k} & 0_{n \times \mathrm{w}}
\end{array}\right] \leq 0,
\end{aligned}
$$

and, there exist $\bar{F}_{k} \in \mathbb{R}^{n_{k} \times n_{k}}$ such that $\widetilde{\Phi}_{k}=$ $\left[\begin{array}{c}\widetilde{X}_{k}^{\top} \\ 0_{\mathrm{w} \times n}\end{array}\right] \bar{F}_{k}\left[\begin{array}{cc}\widetilde{X}_{k} & 0_{n \times \mathrm{w}}\end{array}\right], k=1, \ldots, N$.

Moreover, if for $k, \ell=1, \ldots, N, \ell \neq k$, it holds that

$$
\bar{F}_{k}<0 \text { and } V_{k}^{*} \bar{K}_{k} V_{k} \geq V_{k}^{*} L_{k \rightarrow \ell}^{\top} \bar{K}_{\ell} L_{k \rightarrow \ell} V_{k},
$$

then $\Sigma$ is asymptotically stable, and $\left(X_{k}(\zeta)^{\top} \bar{K}_{k} X_{k}(\eta)\right)_{k=1, \ldots, N}$ induces an MLF.

\section{Proof: See Appendix B.}

Th. 3 reduces the computation of quadratic MLFs as in Th. 1, to the solution of a system of structured LMIs (11)-(12), a straightforward matter for standard LMI solvers.

Remark 4. The fact that no (multiple) quadratic Lyapunov function exists cannot be used to conclude that a system is unstable: the class of quadratic Lyapunov functionals is not universal in the sense of [1], see Corollary 4.3 and Remark 4.1 p. 457 . On this, see also Example 4 below.

The class of polyhedral Lyapunov functions (PLFs) is universal for linear systems with structured uncertainties; in [39] PLFs are applied to linear switched systems in state space form, and a numerical procedure to overcome the complexity of PLF computations is illustrated, see pp. 1021-1022 ibid.

Example 4. The SLDS in Ex. 1 is stable. An MLF is $\left(Q_{\Psi_{1}}, Q_{\Psi_{2}}\right)$, where $\Psi_{1}(\zeta, \eta)=\left[\begin{array}{l}0 \\ 1\end{array}\right]\left[\begin{array}{ll}0 & 1\end{array}\right]=\Psi_{2}(\zeta, \eta)$, inducing the QDFs $Q_{\Psi_{1}}(w)=w_{2}^{2}=Q_{\Psi_{2}}(w)$. Their derivatives along $\mathfrak{B}_{1}$ and $\mathfrak{B}_{2}$ equal $-2 w_{2} \frac{d}{d t} w_{2}=-2 w_{2}^{2}$; due to the gluing conditions, the value of the MLF is the same before and after the switch.

For the system in Ex. 2, straightforward computations show that since the only $R_{i}$-canonical quadratic Lyapunov functionals for $\mathfrak{B}_{i}$ are of the form $\Psi_{i}(\zeta, \eta)=$ $c\left[\begin{array}{l}0 \\ 1\end{array}\right]\left[\begin{array}{ll}0 & 1\end{array}\right], i=1,2$ for $c>0$, no quadratic multiple Lyapunov functions for the SLDS exist. In fact, an argument analogous to that of pp. 126-ff. of [28] proves that the system is unstable.
Remark 5. QDFs act on $\mathfrak{C}^{\infty}$-functions, while trajectories of a SLDS are non-differentiable; however, this mismatch in differentiability is irrelevant to Th. 3 and the other results of this paper. Indeed, we only use the calculus of QDFs as an algebraic tool. For example, in the proof of Th. 3 when considering the value of $Q_{\Psi_{k}}$ and $Q_{\Psi_{\ell}}$ before and after a switch, only the properties of their coefficient matrices are used.

Remark 6. Th. 3 and the associated LMI-based procedure to find an MLF assume that the $\lambda_{k, i}$ and associated directions $w_{k, i}$ are known. If one wants to avoid such pre-computations, a weaker (i.e. more conservative) sufficient condition for the existence of a multiple Lyapunov function can be obtained by solving (11) together with $\bar{F}_{k}<0$ and $\bar{K}_{k} \geq L_{i \rightarrow \ell}^{\top} \bar{K}_{\ell} L_{k \rightarrow \ell}$ in place of (12).

Remark 7. For state-space switched systems, $R_{k}(\xi)=$ $\xi I_{n}-A_{k}$ and $X_{k}(\xi)=I_{n}, k=1, \ldots, N$; straightforward computations yield that in (11) $\bar{Y}_{k}=\bar{K}_{k}$; with the first condition in (12) we obtain the matrix Lyapunov equations $A_{k}^{\top} \bar{K}_{k}+\bar{K}_{k} A_{k}<0$. The second condition in (12) reduces to the classical condition on the reset maps (see e.g. Cor. 2.2 of [15]). For the case of switched DAE's, see sect. 6.3 of [29].

We now illustrate the application of Th. 3 in a realistic setting.

Example 5. Some source converters used in distributed power systems (see e.g. [17]) consist of a traditional DC-DC boost converter coupled with a (dis-)connectable load, see Fig. 2.

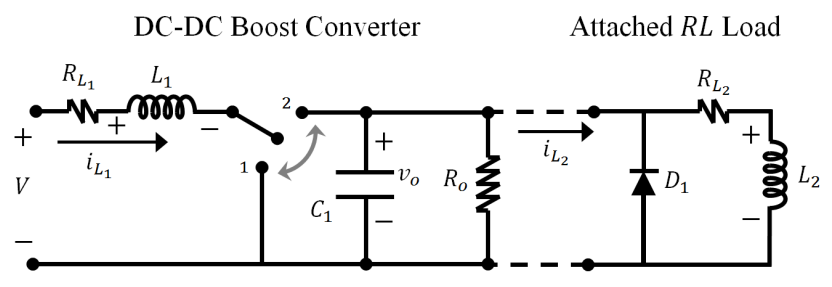

Fig. 2. Source converter.

We take $w=\operatorname{col}\left(i_{L_{1}}, v_{o}\right)$ as the external variable. In order to deal with autonomous behaviours, set the input voltage $V=0$. From standard circuit modelling we conclude that the modes are given by $\mathfrak{B}_{i}=\operatorname{ker} R_{i}\left(\frac{d}{d t}\right)$, $i=1, \ldots, 4$ where

$$
\begin{aligned}
& R_{1}(\xi):=\left[\begin{array}{cc}
L_{1} \xi+R_{L_{1}} & 0 \\
0 & C_{1} \xi+\frac{1}{R_{o}}
\end{array}\right], \\
& R_{2}(\xi):=\left[\begin{array}{cc}
L_{1} \xi+R_{L_{1}} & 1 \\
-1 & C_{1} \xi+\frac{1}{R_{o}}
\end{array}\right],
\end{aligned}
$$




$$
\begin{aligned}
& R_{3}(\xi):= \\
& {\left[\begin{array}{cc}
L_{1} \xi+R_{L_{1}} & 0 \\
0 & L_{2} C_{1} \xi^{2}+\left(R_{L_{2}} C_{1}+\frac{L_{2}}{R_{O}}\right) \xi+\frac{R_{L_{2}}}{R_{O}}+1
\end{array}\right]} \\
& R_{4}(\xi):= \\
& {\left[\begin{array}{cc}
L_{1} \xi+R_{L_{1}} & 1 \\
-L_{2} \xi-R_{L_{2}} & L_{2} C_{1} \xi^{2}+\left(R_{L_{2}} C_{1}+\frac{L_{2}}{R_{o}}\right) \xi+\frac{R_{L_{2}}}{R_{o}}+1
\end{array}\right] .}
\end{aligned}
$$

$\mathfrak{B}_{1}, \mathfrak{B}_{2}$ correspond to the switch in 1 and 2 respectively and the $R L$ load disconnected, and $\mathfrak{B}_{3}, \mathfrak{B}_{4}$ to the modes for the switch in position 1 and 2 and the load connected. The gluing conditions derived from physical considerations are

$$
\begin{aligned}
& \left(I_{2}, I_{2}\right) \\
& =\left(G_{1 \rightarrow 2}^{+}(\xi), G_{1 \rightarrow 2}^{-}(\xi)\right)=\left(G_{2 \rightarrow 1}^{+}(\xi), G_{2 \rightarrow 1}^{-}(\xi)\right) \\
& =\left(G_{3 \rightarrow 1}^{+}(\xi), G_{3 \rightarrow 1}^{-}(\xi)\right)=\left(G_{3 \rightarrow 2}^{+}(\xi), G_{3 \rightarrow 2}^{-}(\xi)\right) \\
& =\left(G_{4 \rightarrow 1}^{+}(\xi), G_{4 \rightarrow 1}^{-}(\xi)\right)=\left(G_{4 \rightarrow 2}^{+}(\xi), G_{4 \rightarrow 2}^{-}(\xi)\right) \text {; } \\
& \begin{aligned}
\left(G_{1 \rightarrow 3}^{+}(\xi), G_{1 \rightarrow 3}^{-}(\xi)\right):= & \left(\left[\begin{array}{cc}
1 & 0 \\
0 & 1 \\
0 & -C_{1} \xi-\frac{1}{R_{o}}
\end{array}\right],\left[\begin{array}{cc}
1 & 0 \\
0 & 1 \\
0 & 0
\end{array}\right]\right) \\
& =:\left(G_{2 \rightarrow 3}^{+}(\xi), G_{2 \rightarrow 3}^{-}(\xi)\right) ;
\end{aligned} \\
& \begin{aligned}
\left(G_{1 \rightarrow 4}^{+}(\xi), G_{1 \rightarrow 4}^{-}(\xi)\right):= & \left(\left[\begin{array}{cc}
1 & 0 \\
0 & 1 \\
1 & -C_{1} \xi-\frac{1}{R_{o}}
\end{array}\right],\left[\begin{array}{ll}
1 & 0 \\
0 & 1 \\
0 & 0
\end{array}\right]\right) \\
& =:\left(G_{2 \rightarrow 4}^{+}(\xi), G_{2 \rightarrow 4}^{-}(\xi)\right) ;
\end{aligned} \\
& \left(G_{3 \rightarrow 4}^{+}(\xi), G_{3 \rightarrow 4}^{-}(\xi)\right)=\left(G_{4 \rightarrow 3}^{-}(\xi), G_{4 \rightarrow 3}^{+}(\xi)\right) \\
& :=\left(\left[\begin{array}{cc}
1 & 0 \\
0 & 1 \\
1 & -C_{1} \xi-\frac{1}{R_{o}}
\end{array}\right],\left[\begin{array}{cc}
1 & 0 \\
0 & 1 \\
0 & -C_{1} \xi-\frac{1}{R_{o}}
\end{array}\right]\right)
\end{aligned}
$$

The following polynomial differential operators induce state maps for $\mathfrak{B}_{k}, k=1, \ldots, 4$ :

$$
\begin{aligned}
& X_{1}(\xi)=X_{2}(\xi):=\left[\begin{array}{ll}
1 & 0 \\
0 & 1
\end{array}\right] ; \\
& X_{3}(\xi):=\left[\begin{array}{cc}
1 & 0 \\
0 & 1 \\
0 & -C_{1} \xi-\frac{1}{R_{o}}
\end{array}\right] ; \\
& X_{4}(\xi):=\left[\begin{array}{cc}
1 & 0 \\
0 & 1 \\
1 & -C_{1} \xi-\frac{1}{R_{o}}
\end{array}\right] .
\end{aligned}
$$

They can be derived by physical considerations or automatically, using the procedures in [22]. Proceeding as in sect. II-B, we compute the re-initialisation maps

$$
\begin{gathered}
L_{1 \rightarrow 2}=L_{2 \rightarrow 1}:=\left[\begin{array}{ll}
1 & 0 \\
0 & 1
\end{array}\right] ; \\
L_{1 \rightarrow 3}=L_{1 \rightarrow 4}=L_{2 \rightarrow 3}=L_{2 \rightarrow 4}:=\left[\begin{array}{ll}
1 & 0 \\
0 & 1 \\
0 & 0
\end{array}\right] ; \\
L_{3 \rightarrow 4}=L_{4 \rightarrow 3}:=I_{3} ; \\
L_{3 \rightarrow 1}=L_{3 \rightarrow 2}=L_{4 \rightarrow 1}=L_{4 \rightarrow 2}:=\left[\begin{array}{lll}
1 & 0 & 0 \\
0 & 1 & 0
\end{array}\right] .
\end{gathered}
$$

With the parameters $L_{1}=100 \mu F, R_{L_{1}}=0.01 \Omega$, $C_{1}=100 \mu F, R_{o}=2 \Omega, R_{L_{2}}=0.02 \Omega, L_{2}=100 \mu F$ we obtain the characteristic frequencies $\lambda_{1,1}=-5000$, $\lambda_{1,2}=-100, \lambda_{2,1}=-2550+j 9695.2=\overline{\lambda_{2,2}}, \lambda_{3,1}=$ $-2600+j 9707.7=\overline{\lambda_{3,2}}, \lambda_{3,3}=-100, \lambda_{4,1}=-149.94$, $\lambda_{4,2}=-2575+j 13933=\overline{\lambda_{4,3}}$. The $V$-matrices of Th. 3 are

$$
V_{1}=\left[\begin{array}{ll}
0 & 1 \\
1 & 0
\end{array}\right]
$$

$$
\begin{gathered}
V_{2}=\left[\begin{array}{cc}
0.70711 & 0.70711 \\
0.17324-j 0.68556 & 0.17324+j 0.68556
\end{array}\right], \\
V_{3}=\left[\begin{array}{ccc}
0 & 0 & 1 \\
0.16971+j 0.68644 & 0.16971-j 0.68644 & 0 \\
0.62564-j 0.32949 & 0.62564+j 0.32949 & 0
\end{array}\right] ;
\end{gathered}
$$

$$
V_{4}=\left[\begin{array}{ccc}
0.70796 & 0.08739+j 0.49199 & 0.08739-j 0.49199 \\
0.00353 & 0.70711 & 0.70711 \\
0.70625 & -0.08407-j 0.49323 & -0.17147+j 0.98522
\end{array}\right] .
$$

Using standard LMI solvers for the LMIs (11), (12) we obtain

$$
\begin{gathered}
\bar{K}_{1}=\bar{K}_{2}=\left[\begin{array}{cc}
0.00123 & -0.00002 \\
-0.00002 & 0.00112
\end{array}\right] ; \\
\bar{K}_{3}=\bar{K}_{4}=\left[\begin{array}{ccc}
0.00123 & -0.00002 & 0 \\
-0.00002 & 0.00112 & 0 \\
0 & 0 & 0.00121
\end{array}\right] .
\end{gathered}
$$

Applying Th. 3 we conclude that $\left(X_{k}(\zeta)^{\top} K_{k} X_{k}(\eta)\right)_{k=1, \ldots, 4}$ induces an MLF.

To illustrate the modularity of our modelling framework, assume that the source converter can also be connected to yet another $R C$ load as depicted in Fig. 3. This results in two additional behaviours in $\mathcal{F}$, namely 


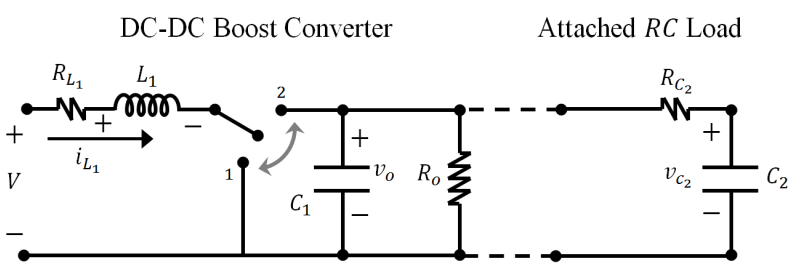

Fig. 3. DC-DC Boost converter/RC-Circuit interconection.

$\mathfrak{B}_{i}=\operatorname{ker} R_{i}\left(\frac{d}{d t}\right), i=5,6$, where

$R_{5}(\xi):=$

$\left[\begin{array}{cc}L_{1} \xi+R_{L_{1}} & 0 \\ 0 & R_{C_{2}} C_{1} C_{2} \xi^{2}+\left(\frac{R_{C_{2}} C_{2}}{R_{o}}+C_{1}+C_{2}\right) \xi+\frac{1}{R_{o}}\end{array}\right]$

$R_{6}(\xi):=$

$\left[\begin{array}{cc}L_{1} \xi+R_{L_{1}} & 1 \\ -R_{C_{2}} C_{2} \xi-1 & R_{C_{2}} C_{1} C_{2} \xi^{2}+\left(\frac{R_{C_{2}} C_{2}}{R_{o}}+C_{1}+C_{2}\right) \xi+\frac{1}{R_{o}}\end{array}\right]$

We choose as state maps for $\mathfrak{B}_{5}$ and $\mathfrak{B}_{6}$

$$
\begin{gathered}
X_{5}(\xi):=\left[\begin{array}{cc}
1 & 0 \\
0 & 1 \\
0 & R_{C_{2}} C_{1} \xi+\frac{R_{C_{2}}}{R_{o}}+1
\end{array}\right] ; \\
X_{6}(\xi):=\left[\begin{array}{cc}
1 & 0 \\
0 & 1 \\
-R_{C_{2}} & R_{C_{2}} C_{1} \xi+\frac{R_{C_{2}}}{R_{o}}+1
\end{array}\right],
\end{gathered}
$$

corresponding to the re-initialisation maps

$$
\begin{gathered}
L_{5 \rightarrow 6}=L_{6 \rightarrow 5}:=I_{3} ; \\
L_{1 \rightarrow 5}=L_{1 \rightarrow 6}=L_{2 \rightarrow 5}=L_{2 \rightarrow 6}:=\left[\begin{array}{ll}
1 & 0 \\
0 & 1 \\
0 & 0
\end{array}\right] ; \\
L_{5 \rightarrow 1}=L_{5 \rightarrow 2}=L_{6 \rightarrow 1}=L_{6 \rightarrow 2}:=\left[\begin{array}{lll}
1 & 0 & 0 \\
0 & 1 & 0
\end{array}\right] .
\end{gathered}
$$

Given the values $R_{C_{2}}=1 \Omega, C_{2}=100 \mu \mathrm{F}$, in order to compute an MLF for $\mathcal{F}:=\left\{\mathfrak{B}_{k}\right\}_{k=1, \ldots, 6}$ we only need to add two LMIs to those set up previously; the solution is

$$
\begin{gathered}
\bar{K}_{1}=\bar{K}_{2}=\left[\begin{array}{cc}
0.00127 & -0.00002 \\
-0.00002 & 0.00126
\end{array}\right] ; \\
\bar{K}_{3}=\bar{K}_{4}=\left[\begin{array}{ccc}
0.00127 & -0.00002 & 0 \\
-0.00002 & 0.00126 & 0 \\
0 & 0 & 0.00131
\end{array}\right] ; \\
\bar{K}_{5}=\bar{K}_{6}=\left[\begin{array}{ccc}
0.00127 & -0.00002 & 0 \\
-0.00002 & 0.00126 & 0 \\
0 & 0 & 0.00382
\end{array}\right] .
\end{gathered}
$$

Remark 8. Ex. 5 illustrates the modularity of our modelling approach for switched systems. Additional dynamic modes (associated with new loads) are incrementally modelled adding them to the existing description. The set of LMIs for the stability analysis is also modularly augmented.

\section{Positive-Realness And Stability of STANDARD SLDS}

The PLE's resemblance to the dissipation equality (see App. A-E) underlies the results of this section, aimed at connecting positive-realness and stability of two-modes SLDS (see [24], [25] in the classical setting). We begin by recalling the definition of strict positivereal rational function (note that this definition is not universally accepted; cf. [27], Th. 2.1.).

Definition 4. $G \in \mathbb{R}^{\mathrm{w} \times \mathrm{w}}(\xi)$ is strictly positive-real if it is analytic in $\mathbb{C}_{+}$and $G(-j \omega)^{\top}+G(j \omega)>0 \forall \omega \in \mathbb{R}$.

We now relate the PLE (9) with strict positive-realness of an associated transfer function.

Proposition 2. Let $N, D \in \mathbb{R}^{\mathrm{w} \times \mathrm{w}}[\xi]$. Assume that $D$ and $N$ are Hurwitz, and that $N D^{-1}$ is strictly proper and strictly positive real. There exist $Q \in \mathbb{R}^{\bullet \times \mathrm{w}}[\xi]$ such that $D(-\xi)^{\top} N(\xi)+N(-\xi)^{\top} D(\xi)=Q(-\xi)^{\top} Q(\xi)$; moreover rank $\operatorname{col}(D(\lambda), Q(\lambda))=\mathrm{w}$ for all $\lambda \in$ $\mathbb{C}$, and $Q D^{-1}$ is strictly proper. Define $\Psi(\zeta, \eta):=$ $\frac{D(\zeta)^{\top} N(\eta)+N(\zeta)^{\top} D(\eta)-Q(\zeta)^{\top} Q(\eta)}{\zeta+\eta}$. Then $\Psi(\zeta, \eta)$ is a $D$ canonical Lyapunov function for $\operatorname{ker} D\left(\frac{d}{d t}\right)$, and $\Psi(\zeta, \eta)$ $\bmod N$ is a Lyapunov function for $\operatorname{ker} N\left(\frac{d}{d t}\right)$.

Proof. See Appendix B.

Thus if $\Psi$ is a suitable storage function of the system with transfer function $N D^{-1}$, associated with a supply rate induced by $\left[\begin{array}{ll}0 & I \\ I & 0\end{array}\right]$ and with dissipation rate $Q(\zeta)^{\top} Q(\eta)$, then it is also a Lyapunov function for ker $D\left(\frac{d}{d t}\right)$ and (after the "mod" operation) also for ker $N\left(\frac{d}{d t}\right)$ (on dissipativity and Lyapunov stability see also [20]). Remarkably, it turns out that such storage functions also induce a $M L F$ for a SLDS with modes $\operatorname{ker} N\left(\frac{d}{d t}\right)$, ker $D\left(\frac{d}{d t}\right)$, and special gluing conditions, naturally associated with the "mod" operation. We now define such systems.

In the following, we consider SLDSs where $\mathcal{F}=$ $\left(\operatorname{ker} R_{1}\left(\frac{d}{d t}\right)\right.$, ker $\left.R_{2}\left(\frac{d}{d t}\right)\right)$, with $R_{j} \in \mathbb{R}^{\mathrm{w} \times \mathrm{w}}[\xi], j=1,2$ nonsingular. We assume that $R_{2} R_{1}^{-1}$ is strictly proper; this implies that the state space of ker $R_{2}\left(\frac{d}{d t}\right)$ is included in that of ker $R_{1}\left(\frac{d}{d t}\right)$, as we presently show. 
Lemma 2. Let $\mathfrak{B}_{i}=\operatorname{ker} R_{i}\left(\frac{d}{d t}\right), i=1,2$. Assume that $R_{1}, R_{2} \in \mathbb{R}^{\mathrm{w} \times \mathrm{w}}[\xi]$ are nonsingular, and that $R_{2} R_{1}^{-1}$ is strictly proper. Let $n_{i}:=\operatorname{deg}\left(\operatorname{det}\left(R_{i}\right)\right)$; then $n_{2}<n_{1}$. There exist $X_{1}^{\prime} \in \mathbb{R}^{\left(n_{1}-n_{2}\right) \times \mathrm{w}}[\xi], X_{2} \in \mathbb{R}^{n_{2} \times \mathrm{w}}[\xi]$ such that $X_{2}\left(\frac{d}{d t}\right)$ is a minimal state map for $\mathfrak{B}_{2}$, and

$$
X_{1}\left(\frac{d}{d t}\right):=\operatorname{col}\left(X_{2}\left(\frac{d}{d t}\right), X_{1}^{\prime}\left(\frac{d}{d t}\right)\right),
$$

is a minimal state map for $\mathfrak{B}_{1}$. Moreover, $\exists \Pi \in$ $\mathbb{R}^{\left(n_{1}-n_{2}\right) \times n_{2}}$ such that $X_{1}^{\prime}(\xi) \bmod R_{2}=\Pi X_{2}(\xi)$.

Proof: See Appendix B.

Example 6. If $\mathrm{w}=1, R_{2} R_{1}^{-1}$ is strictly proper iff $n_{1}=\operatorname{deg}\left(R_{1}\right)>\operatorname{deg}\left(R_{2}\right)=n_{2}$. A state map for $\mathfrak{B}_{1}$ is $\operatorname{col}\left(\xi^{k}\right)_{k=0, \ldots, n_{1}-1}$, whose first $n_{2}$ elements form a basis for the state space of $\mathfrak{B}_{2}$. The rows of $\Pi$ consist of the coefficients of the polynomials $\xi^{k} \bmod R_{2}(\xi)$, $k=n_{2}, \ldots, n_{1}-1$.

In the rest of this section we consider standard SLDS, defined as follows.

Definition 5. Let $\Sigma=\{\mathcal{P}, \mathcal{F}, \mathcal{S}, \mathcal{G}\}$ be a SLDS with $\mathcal{F}=\left(\operatorname{ker} R_{1}\left(\frac{d}{d t}\right), \operatorname{ker} R_{2}\left(\frac{d}{d t}\right)\right)$, where $R_{j} \in \mathbb{R}^{\mathrm{w} \times \mathrm{w}}[\xi]$ is nonsingular, $j=1,2$. Assume that $R_{2} R_{1}^{-1}$ is strictly proper. Let $n_{j}:=\operatorname{deg}\left(\operatorname{det}\left(R_{j}\right)\right)$, $j=1,2$, and let $X_{1}^{\prime} \in \mathbb{R}^{\left(n_{1}-n_{2}\right) \times \mathrm{w}}[\xi]$, $X_{2} \in \mathbb{R}^{n_{2} \times \mathrm{w}}[\xi]$ and $\Pi \in \mathbb{R}^{\left(n_{1}-n_{2}\right) \times n_{2}}$ be as in Lemma 2. $\Sigma$ is a standard SLDS if the gluing conditions are $\left(G_{2 \rightarrow 1}^{-}(\xi), G_{2 \rightarrow 1}^{+}(\xi)\right) \quad:=$ $\left(\operatorname{col}\left(X_{2}(\xi), \Pi X_{2}(\xi)\right), \operatorname{col}\left(X_{2}(\xi), X_{1}^{\prime}(\xi)\right)\right)$ $\left(G_{1 \rightarrow 2}^{-}(\xi), G_{1 \rightarrow 2}^{+}(\xi)\right):=\left(X_{2}(\xi), X_{2}(\xi)\right)$.

Remark 9. It is straightforward to check that the gluing conditions in Def. 5 are well-posed. Note also that the state space of $\mathfrak{B}_{2}$ is contained in that of $\mathfrak{B}_{1}$; however, at any time the state used for the description of the system is that of the active dynamics, and not a global one.

Example 7. Assume that $R_{1}$ and $R_{2}$ in Ex. 6 are monic, and that $n_{1}=n_{2}+1$. Denote $R_{2}(\xi) \quad=: \quad \sum_{j=0}^{n_{1}-1} R_{2, j} \xi^{j}$, and define $S(\xi):=\left[\begin{array}{lll}1 & \ldots & \xi^{n_{1}-2}\end{array}\right]^{\top}$. The gluing conditions of the standard SLDS are $\left(G_{2 \rightarrow 1}^{-}(\xi), G_{2 \rightarrow 1}^{+}(\xi)\right)=$ $\left(\operatorname{col}\left(S(\xi),-\sum_{j=0}^{n_{1}-2} R_{2, j} \xi^{j}\right), \operatorname{col}\left(S(\xi), \xi^{n_{1}-1}\right)\right)$ and $\left(G_{1 \rightarrow 2}^{-}(\xi), G_{1 \rightarrow 2}^{+}(\xi)\right)=(S(\xi), S(\xi))$. In a switch $\mathfrak{B}_{2} \rightarrow \mathfrak{B}_{1}$, to obtain "initial conditions" uniquely specifying $w \in \mathfrak{B}_{1}$, we need to define the value of $\frac{d^{n_{1}-1}}{d t^{n}{ }^{-1}} w$ after the switch. Standard gluing conditions stipulate that it coincides with $\frac{d^{n_{1}-1}}{d t^{n_{1}-1}} w=-\sum_{i=0}^{n_{1}-2} R_{2, i} \frac{d^{i}}{d t^{i}} w$, since before the switch $w \in \mathfrak{B}_{2}$. In a switch $\mathfrak{B}_{1} \rightarrow \mathfrak{B}_{2}$, we project the vector of derivatives characteristic of $w \in \mathfrak{B}_{1}$ down onto the shorter vector of derivatives of $w \in \mathfrak{B}_{2}$.

We now prove that a standard SLDS where $R_{2} R_{1}^{-1}$ is strictly positive real admits a multiple Lyapunov function induced by $\left\{\Psi_{1}, \Psi_{2}\right\}$ where $\Psi_{1}$ is a storage function for $R_{2} R_{1}^{-1}$, and $\Psi_{2}=\Psi_{1} \bmod R_{2}$. This is the multivariable generalisation of some results presented in [23], [21].

Theorem 4. Let $\Sigma$ be a standard SLDS (see Def. 5 ), with $R_{1}$ and $R_{2}$ Hurwitz. Assume that $R_{2} R_{1}^{-1}$ is strictly proper and strictly positive-real. Define $\Phi(\zeta, \eta):=R_{1}(\zeta)^{\top} R_{2}(\eta)+R_{2}(\zeta)^{\top} R_{1}(\eta)$. There exists $Q \in \mathbb{R}^{\bullet \times \mathrm{w}}[\xi]$ such that $\Phi(-\xi, \xi)=Q(-\xi)^{\top} Q(\xi)$, rank $\operatorname{col}\left(R_{1}(\lambda), Q(\lambda)\right)=\mathrm{w}$ for all $\lambda \in \mathbb{C}$ and $Q R_{1}^{-1}$ is strictly proper. Define

$$
\Psi_{1}(\zeta, \eta):=\frac{\Phi(\zeta, \eta)-Q(\zeta)^{\top} Q(\eta)}{\zeta+\eta} .
$$

Then $\Psi_{1}$ is $R_{1}$-canonical. Define $\Psi_{2}:=\Psi_{1} \bmod R_{2}$; then $\left(\Psi_{1}, \Psi_{2}\right)$ induces an MLF for $\Sigma$.

Proof: See Appendix B.

Th. 4 yields two approaches to computing an MLF for a standard SLDS. The first is algebraic and consists of a polynomial spectral factorisation and the computation of $\Psi_{1}$ from (14). The second, based on LMIs, arises from the proof of Th. 4. We state it in the following result.

Corollary 1. Let $X(\xi)$ be a minimal state map for $\mathfrak{B}_{1}$ as in Lemma 2 , and denote by $\widetilde{R}_{1}$ the coefficient matrix of $R_{1}(\xi)$. Under the assumptions of Th. 4 , there exist $\bar{Y} \in$ $\mathbb{R}^{\mathrm{w} \times n_{1}}, \Psi_{11} \in \mathbb{R}^{n_{2} \times n_{2}}$, and $\Psi_{22} \in \mathbb{R}^{\left(n_{1}-n_{2}\right) \times\left(n_{1}-n_{2}\right)}$ such that $\widetilde{\Psi}_{1}:=\left[\begin{array}{cc}\Psi_{11} & -\Pi^{\top} \Psi_{22} \\ -\Psi_{22} \Pi & \Psi_{22}\end{array}\right]>0$ satisfies the LMI

$$
\begin{aligned}
& {\left[\begin{array}{c}
0_{\mathrm{w} \times n_{1}} \\
\widetilde{X}^{\top}
\end{array}\right] \widetilde{\Psi}_{1}\left[\begin{array}{ll}
\widetilde{X} & 0_{n_{1} \times \mathrm{w}}
\end{array}\right]+\left[\begin{array}{c}
\widetilde{X}^{\top} \\
0_{\mathrm{w} \times n_{1}}
\end{array}\right] \widetilde{\Psi}_{1}\left[\begin{array}{ll}
0_{n \times \mathrm{w}} & \widetilde{X}
\end{array}\right]} \\
& -\left[\begin{array}{c}
\widetilde{X}^{\top} \\
0_{\mathrm{w} \times n_{1}}
\end{array}\right] \bar{Y}^{\top} \widetilde{R}_{1}-\widetilde{R}_{1}^{\top} \bar{Y}\left[\begin{array}{ll}
\widetilde{X} & 0_{n_{1} \times \mathrm{w}}
\end{array}\right] \leq 0 .
\end{aligned}
$$

Then $X(\zeta)^{\top} \widetilde{\Psi}_{1} X(\eta)$ and $X_{2}(\zeta)^{\top}\left(\Psi_{11}-\Pi^{\top} \Psi_{22} \Pi\right) X_{2}(\eta) \quad$ induce an $M L F$ for $\Sigma$.

Remark 10. If $\mathrm{w}=1$ the proof of Th. 4 simplifies considerably; see [21] for details.

Remark 11. Theorem 4 holds also if $R_{2} R_{1}^{-1}$ is $b i$ proper, i.e. proper and with a proper inverse; note that in this case the state spaces of $\mathfrak{B}_{1}$ and of $\mathfrak{B}_{2}$ coincide. Let $X \in \mathbb{R}^{\bullet \bullet} \bullet[\xi]$ be a state map for $\mathfrak{B}_{1}$; the standard gluing conditions are $\left(G_{1 \rightarrow 2}^{-}(\xi), G_{1 \rightarrow 2}^{+}(\xi)\right)=(X(\xi), X(\xi))=$ $\left(G_{2 \rightarrow 1}^{-}(\xi), G_{2 \rightarrow 1}^{+}(\xi)\right)$. It is straightforward to check that 
e.g. the largest storage function for $R_{2} R_{1}^{-1}$ yields a MLF. For $\mathrm{w}=1$ this is shown in [23].

Remark 12. In the state-space framework it is wellknown that if the open-loop transfer function of a system is positive-real, then all closed-loop systems obtained from it by state feedback share a common quadratic Lyapunov function (see sect. 2.3.2 of [11] and [24], [25]). Th. 4 offers a new perspective on the relation between positive-realness and stability: in our framework, the different dynamical regimes do not arise from closing the loop around some fixed plant, and positive-realness arises from the interplay of the mode dynamics.

Remark 13. Theorem 4 can also be used to compute from a given Hurwitz matrix $R_{1}$, some matrix $R_{2}$ such that the SLDS with modes ker $R_{i}\left(\frac{d}{d t}\right)$, $i=1,2$ and standard gluing conditions is asymptotically stable. Namely, select $Q \in \mathbb{R}^{\bullet \times \mathrm{w}}[\xi]$ such that rank $\operatorname{col}\left(R_{1}(\lambda), Q(\lambda)\right)=$ w for all $\lambda \in \mathbb{C}$ and $Q R_{1}^{-1}$ is strictly proper; solve the PLE for $R_{2}$. Then the standard SLDS with behaviours ker $R_{i}\left(\frac{d}{d t}\right), i=1,2$ is stable.

For standard SLDS, positive-realness of $R_{2} R_{1}^{-1}$ is a sufficient condition for stability. This assumption is rather restrictive and we now show how to relax it. To this purpose we introduce the concept of positive-real completion.

Definition 6. Let $R_{i} \in \mathbb{R}^{\mathrm{w} \times \mathrm{w}}[\xi], i=1,2$ be nonsingular and $R_{2} R_{1}^{-1}$ strictly proper. $M \in \mathbb{R}^{\mathrm{w} \times \mathrm{w}}[\xi]$ is a strictly positive-real completion of $R_{2} R_{1}^{-1}$ if $M R_{2} R_{1}^{-1}$ is strictly positive-real.

We now show that if an MLF exists, then a positivereal completion can be found.

Theorem 5. Let $\Sigma$ be a standard SLDS (see Def. 5). If $\left\{\Psi_{1}, \Psi_{1} \bmod R_{2}\right\}$ induces an MLF for $\Sigma$ such that $(\zeta+$ $\eta) \Psi_{1}(\zeta, \eta) \bmod R_{1}=-Q(\zeta)^{\top} Q(\eta)$ with rank $Q(j \omega)=$ w for all $\omega \in \mathbb{R}$ and $Q R_{1}^{-1}$ strictly proper, then there exists a strictly positive-real completion $M \in \mathbb{R}^{\mathrm{w} \times \mathrm{w}}[\xi]$ for $R_{2} R_{1}^{-1}$.

\section{Proof: See Appendix.}

Remark 14. An interesting question is whether given a positive-real completion, an MLF induced by $\Psi_{1}$ and $\Psi_{1} \bmod R_{2}$ can be found for some $\Psi_{1} \in \mathbb{R}_{s}^{\mathrm{W} \times \mathrm{W}}[\zeta, \eta]$. The existence of such an MLF can be checked by solving a structured LMI, namely that derived from the positive-real lemma for $M R_{2} R_{1}^{-1}$, together with the structural requirement that the storage function does not increase at the switching instants (see Lemma 3). Such a convex feasibility problem is analogous to those arising in structured Lyapunov problems (see [3]), and can be solved using standard LMI solvers.

\section{Conclusions}

We presented a framework for the modelling and stability analysis of close linear switched systems in which the dynamical modes are not described in statespace form, and do not share a common state space. Pivotal in our approach is the concept of gluing conditions, that impose concatenation constraints on the system trajectories at the switching instants. We devised Lyapunov conditions for general gluing conditions and an arbitrary finite number of modes, amenable to be checked via systems of LMIs. We have also given Lyapunov conditions of a more algebraic flavour based on the concept of positive-realness for two-mode SLDS.

\section{ACKNOWLEDGEMENTS}

The authors gratefully acknowledge their great indebtedness to the Reviewers, who with their many constructive critiques have significantly helped to improve the content and the presentation of the paper.

\section{APPENDIX A}

\section{BACKGROUND MATERIAL}

\section{A. Notation}

The space of $\mathrm{n}$ dimensional real vectors is denoted by $\mathbb{R}^{\mathrm{n}}$, and that of $\mathrm{m} \times \mathrm{n}$ real matrices by $\mathbb{R}^{\mathrm{m} \times \mathrm{n}}$. $\mathbb{R}^{\bullet \times \mathrm{m}}$ denotes the space of real matrices with $m$ columns and an unspecified finite number of rows. Given matrices $A, B \in \mathbb{R}^{\bullet \times \mathrm{m}}, \operatorname{col}(A, B)$ denotes the matrix obtained by stacking $A$ over $B$. The ring of polynomials with real coefficients in the indeterminate $\xi$ is denoted by $\mathbb{R}[\xi]$; the ring of two-variable polynomials with real coefficients in the indeterminates $\zeta$ and $\eta$ is denoted by $\mathbb{R}[\zeta, \eta]$. $\mathbb{R}^{\mathrm{r} \times \mathrm{w}}[\xi]$ denotes the set of all $r \times$ w matrices with entries in $\xi$, and $\mathbb{R}^{\mathrm{n} \times \mathrm{m}}[\zeta, \eta]$ that of $\mathrm{n} \times \mathrm{m}$ polynomial matrices in $\zeta$ and $\eta$. The set of rational $\mathrm{m} \times \mathrm{n}$ matrices is denoted by $\mathbb{R}^{\mathrm{m} \times \mathrm{n}}(\xi)$. We denote by $\bar{\lambda}$ the conjugate of $\lambda \in \mathbb{C}$. The set of infinitely differentiable functions from $\mathbb{R}$ to $\mathbb{R}^{\mathrm{w}}$ is denoted by $\mathfrak{C}^{\infty}\left(\mathbb{R}, \mathbb{R}^{\mathrm{w}}\right)$. If $f$ is a function defined in a neighbourhood $[t-\epsilon, t)$ of $t \in \mathbb{R}$, we set for $f:[t-\epsilon, t) \rightarrow \mathbb{R}^{\bullet}$ the notation $f\left(t^{-}\right):=\lim _{\tau}{ }_{t} f(\tau)$; and similarly for $f:(t, t+\epsilon] \rightarrow \mathbb{R}^{\bullet}$ we set $f\left(t^{+}\right):=$ $\lim _{\tau \searrow t} f(\tau)$, provided that these limits exist.

\section{B. Linear differential behaviours}

$\mathfrak{B} \subseteq \mathfrak{C}^{\infty}\left(\mathbb{R}, \mathbb{R}^{\mathrm{w}}\right)$ is a linear time-invariant differential behaviour if it is the set of solutions of a finite system of constant-coefficient linear differential equations, i.e. 
if there exists $R \in \mathbb{R}^{\mathrm{g} \times \mathrm{w}}[\xi]$ such that $\mathfrak{B}=\{w \in$ $\left.\mathfrak{C}^{\infty}\left(\mathbb{R}, \mathbb{R}^{\mathrm{w}}\right) \mid R\left(\frac{d}{d t}\right) w=0\right\}=$ : ker $R\left(\frac{d}{d t}\right)$. If $\mathfrak{B}=$ ker $R\left(\frac{d}{d t}\right)$, then we call $R$ a kernel representation of $\mathfrak{B}$. We denote by $\mathfrak{L}^{\mathrm{w}}$ the set of all linear time-invariant differential behaviours with w variables. $\mathfrak{B}$ is autonomous if there are no free components in its trajectories; it can be shown that such $\mathfrak{B}$ admits a kernel representation with $R \in \mathbb{R}^{\mathrm{w} \times \mathrm{w}}[\xi]$ square and nonsingular (see [19], Theorem 3.2.16).

Let $R \in \mathbb{R}^{\mathrm{w} \times \mathrm{w}}[\xi]$ be nonsingular, and let $f \in \mathbb{R}^{1 \times \mathrm{w}}[\xi]$; $f R^{-1}$ is uniquely written as $f R^{-1}=s+n$, where $s \in \mathbb{R}^{1 \times \mathrm{w}}(\xi)$ is a vector of strictly proper rational functions, and $n \in \mathbb{R}^{1 \times \mathrm{w}}[\xi]$. We call $s R \in \mathbb{R}^{1 \times \mathrm{w}}[\xi]$ the canonical representative of $f$ modulo $R$, denoted by $f$ $\bmod R$. Note that the polynomial differential operators $f\left(\frac{d}{d t}\right)$ and $f^{\prime}\left(\frac{d}{d t}\right)$, with $f^{\prime}=f \bmod R$, are equivalent along $\operatorname{ker} R\left(\frac{d}{d t}\right)$ in the sense that $f\left(\frac{d}{d t}\right) w=f^{\prime}\left(\frac{d}{d t}\right) w$ for all $w \in \operatorname{ker} R\left(\frac{d}{d t}\right)$. The definition of $R$-canonical representative extends in a natural way to polynomial matrices.

\section{State maps}

A latent variable $\ell$ (see [19], def. 1.3.4 ) is a state variable for $\mathfrak{B}$ iff there exist $E, F \in \mathbb{R}^{\bullet \times \bullet}, G \in \mathbb{R}^{\bullet \times \mathrm{w}}$ such that $\mathfrak{B}=\left\{w \mid \exists \ell\right.$ s.t. $\left.E \frac{d \ell}{d t}+F \ell+G w=0\right\}$, i.e. if $\mathfrak{B}$ has a representation of first order in $\ell$ and zeroth order in $w$. The minimal number of state variables needed to represent $\mathfrak{B}$ in this way is called the McMillan degree of $\mathfrak{B}$, denoted by $\mathrm{n}(\mathfrak{B})$. A state variable for $\mathfrak{B}$ can be computed as the image of a polynomial differential operator called a state map (see [22],[33]). To construct state maps for $\mathfrak{B}:=\operatorname{ker} R\left(\frac{d}{d t}\right)$, with $R \in \mathbb{R}^{\mathrm{w} \times \mathrm{w}}[\xi]$ nonsingular, consider the set $\mathfrak{X}(R):=$ $\left\{f \in \mathbb{R}^{1 \times \mathrm{w}}[\xi] \quad \mid \quad f R^{-1}\right.$ is strictly proper $\} . \mathfrak{X}(R)$ is a finite-dimensional subspace of $\mathbb{R}^{1 \times \mathrm{w}}[\xi]$ over $\mathbb{R}$, (see [22], Prop. 8.4), of dimension $n:=\operatorname{deg}(\operatorname{det}(R)$ ) (see [22], Cor. 6.7). To compute a state map for $\mathfrak{B}$, choose a set of generators $x_{i} \in \mathbb{R}^{1 \times \mathrm{w}}[\xi], i=1, \ldots, N$ of $\mathfrak{X}(R)$, and define $X:=\operatorname{col}\left(x_{i}\right)_{i=1, \ldots, N}$; to obtain a minimal state map, choose $\left\{x_{i}\right\}_{i=1, \ldots, N}$ so that they form a basis of $\mathfrak{X}(R)$. It can be shown that there exists a state map $X$ and $A \in \mathbb{R}^{\bullet \times \bullet}, B \in \mathbb{R}^{\bullet \times \mathrm{w}}$ such that $\xi X(\xi)=A X(\xi)+B R(\xi)$ (see [22], Th. 6.2). Let $\mathfrak{B} \in \mathfrak{L}^{\mathfrak{w}}$, and $X \in \mathbb{R}^{\bullet \times w}[\xi]$ be a state map for $\mathfrak{B}$. A polynomial differential operator $d\left(\frac{d}{d t}\right)$ is a (linear) function of the state of $\mathfrak{B}$ if there exists a constant vector $f \in \mathbb{R}^{1 \times \mathrm{w}}$ such that $d\left(\frac{d}{d t}\right) w=f X\left(\frac{d}{d t}\right) w$ for all $w \in \mathfrak{B}$.

\section{Quadratic differential forms}

Let $\Phi \in \mathbb{R}^{\mathrm{w} \times \mathrm{w}}[\zeta, \eta]$; then $\Phi(\zeta, \eta)=\sum_{h, k} \Phi_{h, k} \zeta^{h} \eta^{k}$, where $\Phi_{h, k} \in \mathbb{R}^{\mathrm{w} \times \mathrm{w}}$ and the sum extends over a finite set of nonnegative indices. $\Phi(\zeta, \eta)$ induces the quadratic differential form (QDF) acting on $\mathfrak{C}^{\infty}$-trajectories defined by $Q_{\Phi}(w):=\sum_{h, k}\left(\frac{d^{h} w}{d t^{h}}\right)^{\top} \Phi_{h, k} \frac{d^{k} w}{d t^{k}}$. Without loss of generality QDF is induced by a symmetric twovariable polynomial matrix $\Phi(\zeta, \eta)$, i.e. one such that $\Phi(\zeta, \eta)=\Phi(\eta, \zeta)^{\top}$; we denote the set of such matrices by $\mathbb{R}_{S}^{\mathrm{W} \times \mathrm{W}}[\zeta, \eta]$. Given $Q_{\Psi}$, its derivative is the QDF $Q_{\Phi}$ defined by $Q_{\Phi}(w):=\frac{d}{d t}\left(Q_{\Psi}(w)\right)$ for all $w \in \mathfrak{C}^{\infty}\left(\mathbb{R}, \mathbb{R}^{\mathrm{w}}\right)$; this holds if and only if $\Phi(\zeta, \eta)=$ $(\zeta+\eta) \Psi(\zeta, \eta)$ (see [36], p. 1710). $Q_{\Phi}$ is nonnegative along $\mathfrak{B} \in \mathfrak{L}^{\mathrm{w}}$, denoted by $Q_{\Phi} \stackrel{\mathfrak{B}}{\geq} 0$ if $Q_{\Phi}(w) \geq 0$ for all $w \in \mathfrak{B}$; and positive along $\mathfrak{B}$, denoted by $Q_{\Phi} \stackrel{\mathfrak{B}}{>} 0$, if $Q_{\Phi} \stackrel{\mathfrak{B}}{\geq} 0$ and $\left[Q_{\Phi}(w)=0 \forall w \in \mathfrak{B}\right] \Longrightarrow[w=0]$. If $\mathfrak{B}=\mathfrak{C}^{\infty}\left(\mathbb{R}, \mathbb{R}^{\mathrm{w}}\right)$, then we call $Q_{\Phi}$ simply nonnegative, respectively positive. For algebraic characterizations of these properties see [36], pp. 1712-1713. Let $R \in$ $\mathbb{R}^{\mathrm{w} \times \mathrm{w}}[\xi]$ be nonsingular and $\Phi \in \mathbb{R}^{\mathrm{w} \times \mathrm{w}}[\zeta, \eta]$. Factorise $\Phi(\zeta, \eta)=M(\zeta)^{\top} N(\eta)$ and compute the $R$-canonical representatives (see App. A-B) $M^{\prime}=M \bmod R$ and $N^{\prime}=N \bmod R$. The $R$-canonical representative of $\Phi(\zeta, \eta)$ is defined by $\Phi(\zeta, \eta) \bmod R:=M^{\prime}(\zeta)^{\top} N^{\prime}(\eta)$. The QDFs $Q_{\Phi}, Q_{\Phi^{\prime}}$ are equivalent along $\operatorname{ker} R\left(\frac{d}{d t}\right)$, i.e. $Q_{\Phi^{\prime}}(w)=Q_{\Phi}(w)$ for all $w \in \operatorname{ker} R\left(\frac{d}{d t}\right)$.

\section{E. Dissipativity}

A controllable (see Ch. 5 of [19]) behaviour $\mathfrak{B} \in \mathfrak{L}^{\text {w }}$ is dissipative with respect to the supply rate $Q_{\Phi}$ if there exists a QDF $Q_{\Psi}$, called a storage function, such that $Q_{\Phi}(w)-\frac{d}{d t} Q_{\Psi}(w) \geq 0$ for all $w \in \mathfrak{B}$. This inequality holds iff there exists a dissipation function, i.e. a QDF $Q_{\Delta} \stackrel{\mathfrak{B}}{\geq} 0$ such that for all $w \in \mathfrak{B}$ of compact support it holds that $\int_{-\infty}^{+\infty} Q_{\Phi}(w)(t) d t=\int_{-\infty}^{+\infty} Q_{\Delta}(w)(t) d t$ (see Prop. 5.4 of [36]). Moreover, there is a one-one correspondence between storage- and dissipation functions, defined by $\frac{d}{d t} Q_{\Psi}(w)+Q_{\Delta}(w)=Q_{\Phi}(w)$ for all $w \in \mathfrak{B}$. If $\mathfrak{B}=\mathfrak{C}^{\infty}\left(\mathbb{R}, \mathbb{R}^{\mathrm{w}}\right)$, this equality holds if and only if $(\zeta+\eta) \Psi(\zeta, \eta)+\Delta(\zeta, \eta)=\Phi(\zeta, \eta)$.

\section{APPENDIX B PROOFS}

Proof of Th. 1: Let $s \in \mathcal{S}$ be a switching signal, and from $\left\{Q_{\Psi_{1}}, \ldots, Q_{\Psi_{N}}\right\}$ define the "switched functional" $Q_{\Lambda}$ acting on $\mathfrak{B}^{\Sigma}$ by $Q_{\Lambda}(w)(t):=Q_{\Psi_{s(t)}}(w)(t)$. Observe that in every interval $\left[t_{j-1}, t_{j}\right) Q_{\Lambda}$ is nonnegative, continuous and strictly decreasing, since $Q_{\Psi_{s\left(t_{j-1}\right)}}$ satisfies conditions 1) - 2). Moreover, for every admissible trajectory the value of $Q_{\Lambda}$ does not increase at switching instants (condition 3)). It follows from standard arguments (see e.g. Th. 4.1 of [38]) that $\Sigma$ is asymptotically stable. 
Proof of Lemma 1: The existence of $\bar{K}=\bar{K}^{\top} \in$ $\mathbb{R}^{n \times n}, \bar{Y} \in \mathbb{R}^{\mathrm{w} \times n}, \bar{Q} \in \mathbb{R}^{\bullet \times n}$ follows from Th. 2 and the fact that the rows of $X(\xi)$ are a basis for the vector space over $\mathbb{R}$ defined by $\left\{f \in \mathbb{R}^{1 \times \mathrm{w}}[\xi] \mid\right.$ $f R^{-1}$ is strictly proper $\}$. The fact that the degree of $X(\xi)$ is less than that of $R(\xi)$ follows from $X R^{-1}$ being strictly proper and Lemma 6.3-10 of [10].

\section{Proof of Prop. 1:}

In order to prove the equivalence of statements 1 . and 2 , define $S_{L}(\xi):=\left[\begin{array}{llll}I_{\mathrm{w}} & \xi I_{\mathrm{w}} & \ldots & \xi^{L} I_{\mathrm{w}}\end{array}\right]^{\top}$; the equivalence follows in a straightforward way from the first part of the claim and the equalities $X(\xi)=\left[\begin{array}{llll}X_{0} & \ldots & X_{L-1} & 0_{n \times \mathrm{w}}\end{array}\right] S_{L}(\xi)$, $\xi X(\xi)=\left[\begin{array}{llll}0_{n \times \mathrm{w}} & X_{0} & \ldots & X_{L-1}\end{array}\right] S_{L}(\xi), \quad$ and $R(\xi)=\left[\begin{array}{llll}R_{0} & \ldots & R_{L-1} & R_{L}\end{array}\right] S_{L}(\xi)$. The final part of the claim follows in straightforward way.

Proof of Th. 3: Solutions $\bar{K}_{k}, \bar{Y}_{k}$ to (11) exist because of Th. 2, Lemma 1 and Prop. 1. Multiply (11) on the left by $S_{L}(\zeta)^{\top}$ defined as in the proof of Prop. 1 and on the right by $S_{L}(\eta)$, and define $\Psi_{k}(\zeta, \eta):=$ $X_{k}(\zeta)^{\top} \bar{K}_{k} X_{k}(\eta)$ and $Y_{k}(\xi):=\bar{Y}_{k} X_{k}(\xi)$ to obtain $(\zeta+\eta) \Psi_{k}(\zeta, \eta)-Y_{k}(\zeta)^{\top} R_{k}(\eta)-R_{k}(\zeta)^{\top} Y_{k}(\eta)=$ $\Phi_{k}(\zeta, \eta)$. Since $Y_{k}$ is $R$-canonical, it follows from Th. 2 that also $\Phi_{k}(\zeta, \eta)$ is, and consequently $\bar{F}_{k}$ exist as claimed. Now observe that the first inequality in (12) is equivalent with $V_{k}^{\top} \bar{F}_{k} V_{k}<0$ and thus it implies $Q_{\Phi_{k}}(w)=\frac{d}{d t} Q_{\Psi_{k}}(w)<0$ for all $w \in \mathfrak{B}_{k}$. Applying Th. 2 we conclude that $Q_{\Psi_{k}}$ is a Lyapunov function for $\mathfrak{B}_{k}$. The second LMI in (12) implies condition 3. of Th. 1.

Proof of Prop. 2: From the strict positive-realness of $N D^{-1}$ (see Def. 4) and the fact that $D$ is Hurwitz conclude that $N(-j \omega)^{\top} D(j \omega)+D(-j \omega)^{\top} N(j \omega)>0$ for all $\omega \in \mathbb{R}$. The existence of $Q$ then follows from standard arguments in polynomial spectral factorisation. That $\Psi$ is a polynomial matrix follows from Th. 3.1 of [36]. Since rank $\operatorname{col}(D(\lambda), Q(\lambda))=$ w for all $\lambda \in \mathbb{C}$, $\frac{d}{d t} Q_{\Psi}(w)<0$ for all $w \in \operatorname{ker} D\left(\frac{d}{d t}\right), w \neq 0$. Apply Th. 2 to conclude that $Q_{\Psi}(w)>0$ for all nonzero $w \in$ $\operatorname{ker} D\left(\frac{d}{d t}\right)$. This proves that $\Psi$ is a Lyapunov function for $\operatorname{ker} D\left(\frac{d}{d t}\right)$. That $\Psi$ is $D$-canonical and $Q D^{-1}$ strictly proper, follow from strict properness of $N D^{-1}$ and $\mathrm{Th}$. 2.

We prove the second part of the claim. Use Prop. 4.10 of [36] to conclude that since $\Psi$ is $D$-canonical, it is also $\geq 0$. Denote $\Psi^{\prime}:=\Psi \bmod N$. Since $Q_{\Psi}(w)=Q_{\Psi^{\prime}}(w)$ for all $w \in \operatorname{ker} N\left(\frac{d}{d t}\right)$, it follows that $Q_{\Psi^{\prime}} \geq 0$ also along ker $N\left(\frac{d}{d t}\right)$. We now show that $\frac{d}{d t} Q_{\Psi^{\prime}}$ is negative along ker $N\left(\frac{d}{d t}\right)$. To do so it suffices to show that $\operatorname{col}(Q(\lambda), N(\lambda))=$ w for all $\lambda \in \mathbb{C}$. Assume by contradiction that there exists $\lambda \in \mathbb{C}$ and a corresponding $v \in \mathbb{C}^{\mathrm{w}}, v \neq 0$, such that $Q(\lambda) v=0$ and $N(\lambda) v=0$.
Substitute $\zeta=-\lambda, \eta=\lambda$ in the PLE, obtaining $D(-\lambda)^{\top} N(\lambda)+N(-\lambda)^{\top} D(\lambda)=Q(-\lambda)^{\top} Q(\lambda)$. Multiply on the right by $v$; it follows that $N(-\lambda)^{\top} D(\lambda) v=0$. Since $N$ is Hurwitz, this implies $D(\lambda) v=0$, but this contradicts the assumption $\operatorname{rank} \operatorname{col}(D(\lambda), Q(\lambda))=$ w.

Proof of Lemma 2: That $n_{2}<n_{1}$ follows from $R_{2} R_{1}^{-1}$ being strictly proper.

To prove the claim on $X_{1}$ defined by (13), define $\mathfrak{X}_{i}:=\left\{f \in \mathbb{R}^{1 \times \mathrm{w}}[\xi] \mid f R_{i}^{-1}\right.$ is strictly proper $\}$, $i=1,2$; we now show that $\mathfrak{X}_{2} \subset \mathfrak{X}_{1}$. Observe that $f R_{2}^{-1} \cdot R_{2} R_{1}^{-1}=f R_{1}^{-1}$; since both $f R_{2}^{-1}$ and $R_{2} R_{1}^{-1}$ are strictly proper, so is their product. Consequently, $f \in \mathfrak{X}_{1}$. Observe that $\mathfrak{X}_{i}$ is the state space of $\mathfrak{B}_{i}, i=1,2$ (see App. A-C). Arrange the vectors of a basis for $\mathfrak{X}_{2}$ in $X_{2} \in \mathbb{R}^{n_{2} \times \mathrm{w}}[\xi]$; then $X_{2}\left(\frac{d}{d t}\right)$ is a state map for $\mathfrak{B}_{2}$. Complete $X_{2}$ with $X_{1}^{\prime} \in \mathbb{R}^{\left(n_{1}-n_{2}\right) \times \mathrm{w}}[\xi]$ to form a basis of $\mathfrak{X}_{1}$; this defines a state map for $\mathfrak{B}_{1}$. Since each row of $X_{1}^{\prime} \bmod R_{2}$ belongs to $\mathfrak{X}_{2}$, it can be written as a linear combination of the rows of $X_{2}$. This proves that $\Pi$ exists.

Proof of Theorem 4: The existence of $Q \in$ $\mathbb{R}^{\bullet \times \mathrm{w}}[\xi]$ and the $R_{1}$-canonicity of $\Psi_{1}$ follow from Prop. 2. To prove that $\Psi_{1}$ and $\Psi_{2}:=\Psi_{1} \bmod R_{2}$ yield an MLF we show that:

C1. $Q_{\Psi_{1}} \stackrel{\mathfrak{B}_{1}}{\geq} 0$ and $\frac{d}{d t} Q_{\Psi_{1}} \stackrel{\mathfrak{B}_{1}}{<} 0$;

C2. $Q_{\Psi_{2}} \stackrel{\mathfrak{B}_{2}}{\geq} 0$ and $\frac{d}{d t} Q_{\Psi_{2}} \stackrel{\mathfrak{B}_{2}}{<} 0$;

C3. The multiple functional associated with $\Psi_{1}$ and $\Psi_{2}$ does not increase at switching instants.

Conditions C1 and C2 follow from Prop. 2. To prove $\mathbf{C 3}$, we first define the coefficient matrices of $\Psi_{1}$ and $\Psi_{2}$. Since $\Psi_{1}$ is $R_{1}$-canonical, it can be written as $X_{1}(\zeta)^{\top} \widetilde{\Psi}_{1} X_{1}(\eta)$ for some coefficient matrix $\widetilde{\Psi}_{1} \in \mathbb{R}^{n_{1} \times n_{1}}$. Since $Q R_{1}^{-1}$ is strictly proper, it follows (see Th. 2) that $Q_{\Psi_{1}} \stackrel{\mathfrak{B}}{>} 0$ and since $X_{1}$ is a minimal state map for $\mathfrak{B}_{1}$ it follows that $\widetilde{\Psi}_{1}>0$. Note that $\operatorname{col}\left(X_{2}(\xi), X_{1}^{\prime}(\xi)\right) \bmod R_{2}=$ $\operatorname{col}\left(X_{2}(\xi) \quad \bmod R_{2}, X_{1}^{\prime}(\xi) \quad \bmod \quad R_{2}\right)=$ $\operatorname{col}\left(X_{2}(\xi), \Pi X_{2}(\xi)\right) . \quad$ Consequently $\quad$ (see Prop. 4.9 of [36]), $\quad \Psi_{1}(\zeta, \eta) \bmod R_{2}=$

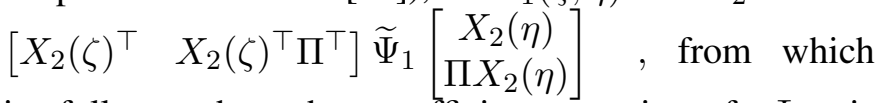
it follows that the coefficient matrix of $\Psi_{2}$ is $\widetilde{\Psi}_{2}=\operatorname{col}\left(I_{n_{2}}, \Pi\right)^{\top} \widetilde{\Psi}_{1} \operatorname{col}\left(I_{n_{2}}, \Pi\right)$.

We prove $\mathbf{C 3}$ showing that $\widetilde{\Psi}_{1}$ and $\widetilde{\Psi}_{2}$ satisfy some structural properties. We begin proving the following linear algebra result.

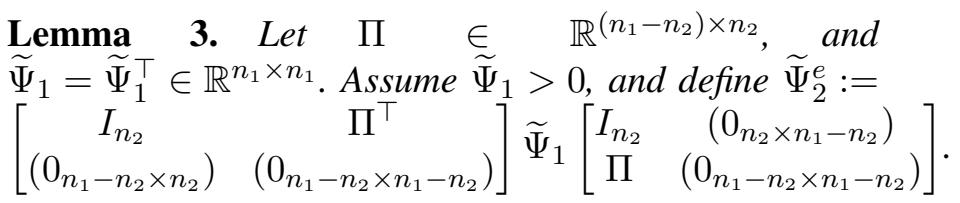


$\widetilde{\Psi}_{1} \geq \widetilde{\Psi}_{2}^{e}$ if and only if there exist $\Psi_{11} \in \mathbb{R}^{n_{2} \times n_{2}}$, $\Psi_{12} \in \mathbb{R}^{n_{2} \times\left(n_{1}-n_{2}\right)}$ and $\Psi_{22} \in \mathbb{R}^{\left(n_{1}-n_{2}\right) \times\left(n_{1}-n_{2}\right)}$ such that $\widetilde{\Psi}_{1}=\left[\begin{array}{cc}\Psi_{11} & -\Pi^{\top} \Psi_{22} \\ -\Psi_{22} \Pi & \Psi_{22}\end{array}\right]$.

Proof of Lemma 3: Partition $\widetilde{\Psi}_{1}=:\left[\begin{array}{ll}\Psi_{11} & \Psi_{12} \\ \Psi_{12}^{\top} & \Psi_{22}\end{array}\right]$, with $\Psi_{11} \in \mathbb{R}^{n_{2} \times n_{2}}, \Psi_{12} \in \mathbb{R}^{n_{2} \times\left(n_{1}-n_{2}\right)}$ and $\Psi_{22} \in$ $\mathbb{R}^{\left(n_{1}-n_{2}\right) \times\left(n_{1}-n_{2}\right)}$. Straightforward manipulations show that $\widetilde{\Psi}_{1} \geq \widetilde{\Psi}_{2}^{e}$ iff

$$
\left[\begin{array}{cc}
-\left(\Psi_{12}+\Pi^{\top} \Psi_{22}\right) \Psi_{22}^{-1}\left(\Psi_{12}^{\top}+\Psi_{22} \Pi\right) & 0 \\
0 & \Psi_{22}
\end{array}\right] \geq 0 .
$$

Now $\Psi_{22}>0$, since $\widetilde{\Psi}_{1}>0$; thus the inequality holds iff $\Psi_{12}^{\top}=-\Psi_{22} \Pi$.

We aim to show that Lemma 3 holds for the coefficient matrix of $\Psi_{1}$ and the $\Pi$ arising from the standard gluing conditions. To this purpose we first prove the following result.

Lemma 4. Define $K:=\lim _{\xi \rightarrow \infty} \xi X_{1}^{\prime}(\xi) R_{1}(\xi)^{-1}$; then $K \in \mathbb{R}^{\left(n_{1}-n_{2}\right) \times \mathrm{w}}$. Moreover, partition $\widetilde{\Psi}_{1}$ as $\widetilde{\Psi}_{1}=:\left[\begin{array}{ll}\Psi_{11} & \Psi_{12} \\ \Psi_{12}^{\top} & \Psi_{22}\end{array}\right]$, with $\Psi_{11} \in \mathbb{R}^{n_{2} \times n_{2}}, \Psi_{12} \in$ $\mathbb{R}^{n_{2} \times\left(n_{1}-n_{2}\right)}$ and $\Psi_{22} \in \mathbb{R}^{\left(n_{1}-n_{2}\right) \times\left(n_{1}-n_{2}\right)}$. Then $R_{2}(\xi)=K^{\top}\left(\Psi_{12}^{\top} X_{2}(\xi)+\Psi_{22} X_{1}^{\prime}(\xi)\right)$.

Proof of Lemma 4: That the limit is finite follows from $X_{1}^{\prime} R_{1}^{-1}$ being strictly proper. To prove the rest, recall from App. A-C that there exist $A_{1} \in \mathbb{R}^{n_{1} \times n_{1}}$, $F_{1} \in \mathbb{R}^{n_{1} \times \text { w }}$ such that

$$
\xi X_{1}(\xi)=A_{1} X_{1}(\xi)+F_{1}(\xi) R_{1}(\xi) .
$$

Multiply both sides of (15) by $R_{1}^{-1}$, and take the limit for $\xi \rightarrow \infty$. Since $R_{2} R_{1}^{-1}$ is strictly proper and $X_{2}(\xi)$ is a state map for $\mathfrak{B}_{2}$, it follows that $\lim _{\xi \rightarrow \infty} \xi X_{2}(\xi) R_{1}(\xi)^{-1}=0_{n_{2} \times \mathrm{w}}$. Moreover, $\lim _{\xi \rightarrow \infty} X_{1}(\xi) R_{1}(\xi)^{-1}=0_{n_{1} \times \mathrm{w}}$. Consequently $F_{1}$ is constant, and

$F_{1}=\lim _{\xi \rightarrow \infty} \operatorname{col}\left(0_{n_{2} \times \mathrm{w}}, \xi X_{1}^{\prime}(\xi) R_{1}(\xi)^{-1}\right)=\operatorname{col}\left(0_{n_{2} \times \mathrm{w}}, K\right)$.

The claim on $R_{2}$ now follows from Prop. 4.3 of [16].

From Lemma 4 and the fact that $R_{2}$ is square and nonsingular, it follows that $K^{\top}$ is of full row rank, and consequently $n_{1}-n_{2} \geq$ w. We now prove that $K$ is square, thus nonsingular.

Lemma 5. $\operatorname{deg}\left(\operatorname{det}\left(R_{1}\right)\right)-\operatorname{deg}\left(\operatorname{det}\left(R_{2}\right)\right)=n_{1}-n_{2}=\mathrm{w}$, and consequently $K$ is nonsingular.

Proof of Lemma 5: We prove the first part of the claim, well-known in the scalar case, but for whose multivariable version we have failed to find a proof in the literature.
Let $U \in \mathbb{R}^{\mathrm{w} \times \mathrm{w}}[\xi]$ be a unimodular matrix such that $R_{1}^{\prime}:=R_{1} U$ is column reduced (see sect. 6.3.2 of [10]); define $R_{2}^{\prime}:=R_{2} U$. Observe that $R_{2}^{\prime} R_{1}^{\prime-1}=R_{2} R_{1}^{-1}$; moreover $n_{1}=\operatorname{deg}\left(\operatorname{det}\left(R_{1}^{\prime}\right)\right)=\operatorname{deg}\left(\operatorname{det}\left(R_{1}\right)\right)$ and $n_{2}=\operatorname{deg}\left(\operatorname{det}\left(R_{2}\right)\right)=\operatorname{deg}\left(\operatorname{det}\left(R_{2}^{\prime}\right)\right)$. Thus w.l.o.g. we prove the claim for $R_{2}^{\prime} R_{1}^{\prime-1}$. Define $\mathfrak{X}_{1}^{\prime}:=\{f \in$ $\mathbb{R}^{1 \times \mathrm{w}}[\xi] \mid f R_{1}^{\prime-1}$ is strictly proper $\}$ and similarly $\mathfrak{X}_{2}^{\prime}$; it is straightforward to see that $\mathfrak{X}_{i}^{\prime}$ equals $\mathfrak{X}_{i}$ defined as in Lemma $2, i=1,2$. Denote the degree of the $i$ th column of $R_{1}^{\prime}$ by $\delta_{i}^{1}$ and that of the $i$-th column of $R_{2}^{\prime}$ by $\delta_{i}^{2}, i=1, \ldots$, w; strict properness yields $\delta_{i}^{1}>\delta_{i}^{2}, i=1, \ldots$, w. A basis for $\mathfrak{X}_{1}^{\prime}$ is $e_{i} \xi^{k}$, $k=1, \ldots, \delta_{k}^{1}-1, i=1, \ldots, \mathrm{w}$, where $e_{i}$ is the $i$-th vector of the canonical basis for $\mathbb{R}^{1 \times \mathrm{w}}$. A straightforward argument proves that these vectors can be arranged in a matrix $X(\xi)=\operatorname{col}\left(X_{2}(\xi), X_{1}^{\prime}(\xi)\right)$ so that the $n_{2}$ rows of $X_{2}$ span $\mathfrak{X}_{2}^{\prime}$ and those of $X_{1}^{\prime}$ span its complement in $\mathfrak{X}_{1}^{\prime}$. Permute the rows of $X_{1}^{\prime}$ so that $e_{i} \xi^{\delta_{i}^{1}-1}, i=1, \ldots, \mathrm{w}$, are its last w rows.

An analogous of (15) holds for $R_{1}^{\prime}$; given the arrangement of the basis vectors for $\mathfrak{X}_{1}^{\prime}$, it is straightforward to verify that the last w rows of $K$ contain the inverse of the highest column coefficient matrix of $R_{1}$, while its first $n_{1}-n_{2}-\mathrm{w}$ rows are equal to zero, i.e. $K^{\top}=$ $\left[\begin{array}{ll}0_{\left(n_{1}-n_{2}-\mathrm{w}\right) \times \mathrm{w}} & K^{\prime \top}\end{array}\right]$, with $K^{\prime} \in \mathbb{R}^{\mathrm{w} \times \mathrm{w}}$ nonsingular.

Now let $\Psi_{1}^{\prime}$ be a storage function for $R_{2}^{\prime} R_{1}^{\prime-1}$ with the same properties as $\Psi_{1}$ in the statement of Th. 4; we denote with $\Psi_{i j}^{\prime}, i, j=1,2$ the block submatrices arising from a partition of its coefficient matrix $\widetilde{\Psi^{\prime}}{ }_{1}$ as in Lemma 4 . Use the formula for $R_{2}^{\prime}(\xi)$ established in Lemma 4 to conclude that $R_{2}^{\prime}(\xi)=$ $K^{\prime \top} \Psi_{12}^{\prime \top} X_{2}(\xi)+K^{\prime \top}\left[\Psi_{22}^{\prime \prime} \quad \Psi_{22}^{\prime \prime \prime}\right] X_{1}^{\prime}(\xi)$, where $\Psi_{12}^{\prime \top} \in$ $\mathbb{R}^{\mathrm{w} \times n_{2}}, \quad\left[\begin{array}{ll}\Psi_{22}^{\prime \prime} & \Psi_{22}^{\prime \prime \prime}\end{array}\right] \in \mathbb{R}^{\mathrm{w} \times\left(n_{1}-n_{2}\right)}$, and $\Psi_{22}^{\prime \prime \prime}$ has $\mathrm{w}$ columns. $\Psi_{1}^{\prime}>0$ implies $\Psi_{22}^{\prime \prime \prime}>0$; thus the highest column coefficient matrix of $R_{2}(\xi)$ is $K^{\prime} \Psi_{22}^{\prime \prime \prime}$ and it is nonsingular. Thus also $R_{2}^{\prime}(\xi)$ is column reduced; moreover, its column degrees are $\delta_{i}^{1}-1, i=1, \ldots$, w. From this it follows that $\operatorname{deg} \operatorname{det}\left(R_{2}^{\prime}\right)=\sum_{i=1}^{\mathrm{w}}\left(\delta_{i}^{1}-1\right)=$ $\left(\sum_{i=1}^{\mathrm{W}} \delta_{i}^{1}\right)-\mathrm{w}=n_{1}-\mathrm{w}$. The claim is proved.

We resume the proof of Th. 4. From the formula for $R_{2}(\xi)$ proved in Lemma 4 it follows that

$$
\begin{aligned}
0 & =R_{2}(\xi) \bmod R_{2} \\
& =K^{\top}\left(\Psi_{12}^{\top} X_{2}(\xi)+\Psi_{22} X_{1}^{\prime}(\xi)\right) \bmod R_{2} \\
& =K^{\top}\left(\Psi_{12}^{\top}+\Psi_{22} \Pi\right) X_{2}(\xi) .
\end{aligned}
$$

The rows of $X_{2}(\xi)$ are linearly independent over $\mathbb{R}$, since $X_{2}$ is a minimal state map. Consequently (16) implies $K^{\top}\left(\Psi_{12}^{\top}+\Psi_{22} \Pi\right)=0$, and since $K$ is nonsingular by Lemma 5, we conclude that $\Psi_{12}^{\top}+\Psi_{22} \Pi=0$. Thus the coefficient matrix of $\Psi_{1}$ is structured as in Lemma 3. 
We now show that this structure implies that condition C3 holds. Consider first a switch from $\mathfrak{B}_{1}$ to $\mathfrak{B}_{2}$ at $t_{k}$. Taking the standard gluing conditions into account, $Q_{\Psi_{1}}(w)\left(t_{k}^{-}\right) \geq Q_{\Psi_{2}}(w)\left(t_{k}^{+}\right)$if and only if

$$
\begin{aligned}
& {\left[\begin{array}{l}
X_{2}\left(\frac{d}{d t}\right) w\left(t_{k}^{-}\right) \\
X_{1}^{\prime}\left(\frac{d}{d t}\right) w\left(t_{k}^{-}\right)
\end{array}\right]^{\top} \widetilde{\Psi}_{1}\left[\begin{array}{l}
X_{2}\left(\frac{d}{d t}\right) w\left(t_{k}^{-}\right) \\
X_{1}^{\prime}\left(\frac{d}{d t}\right) w\left(t_{k}^{-}\right)
\end{array}\right]} \\
& -\left[\begin{array}{c}
X_{2}\left(\frac{d}{d t}\right) w\left(t_{k}^{+}\right) \\
\Pi X_{2}\left(\frac{d}{d t}\right) w\left(t_{k}^{+}\right)
\end{array}\right]^{\top} \widetilde{\Psi}_{1}\left[\begin{array}{c}
X_{2}\left(\frac{d}{d t}\right) w\left(t_{k}^{+}\right) \\
\Pi X_{2}\left(\frac{d}{d t}\right) w\left(t_{k}^{+}\right)
\end{array}\right] \\
& =\left[\begin{array}{l}
X_{2}\left(\frac{d}{d t}\right) w\left(t_{k}^{-}\right) \\
X_{1}^{\prime}\left(\frac{d}{d t}\right) w\left(t_{k}^{-}\right)
\end{array}\right]^{\top}\left(\widetilde{\Psi}_{1}-\left[\begin{array}{cc}
I_{n_{2}} & \Pi^{\top} \\
0 & 0
\end{array}\right] \widetilde{\Psi}_{1}\left[\begin{array}{cc}
I_{n_{2}} & 0 \\
\Pi & 0
\end{array}\right]\right) \\
& \\
& {\left[\begin{array}{l}
X_{2}\left(\frac{d}{d t}\right) w\left(t_{k}^{-}\right) \\
X_{1}^{\prime}\left(\frac{d}{d t}\right) w\left(t_{k}^{-}\right)
\end{array}\right] \geq 0 .}
\end{aligned}
$$

Since the matrix between brackets is semidefinite positive (see Lemma 3), (17) is satisfied.

It is straightforward to check that in a switch from $\mathfrak{B}_{2}$ to $\mathfrak{B}_{1}$ the value of the multi-functional is the same before and after the switch. The theorem is proved.

Proof of Th. 5: W.1.o.g. assume that $Q_{\Psi}$ is $R_{1}$ canonical; then by Lemma 2, given a minimal state map $X_{1}\left(\frac{d}{d t}\right)$ for $\mathfrak{B}_{1}$ as in (13) there exists $\widetilde{\Psi}=$ $\widetilde{\Psi}^{\top} \in \mathbb{R}^{n_{1} \times n_{1}}$ such that $\Psi(\zeta, \eta)=X_{1}(\zeta)^{\top} \widetilde{\Psi} X_{1}(\eta)$. Partition $\widetilde{\Psi}$ as $\widetilde{\Psi}=:\left[\begin{array}{ll}\Psi_{11} & \Psi_{12} \\ \Psi_{12}^{\top} & \Psi_{22}\end{array}\right]$ where $\Psi_{11} \in \mathbb{R}^{n_{2} \times n_{2}}$, $\Psi_{12} \in \mathbb{R}^{n_{2} \times\left(n_{1}-n_{2}\right)}$ and $\Psi_{22} \in \mathbb{R}^{\left(n_{1}-n_{2}\right) \times\left(n_{1}-n_{2}\right)}$. At a switch from $\mathfrak{B}_{1}$ to $\mathfrak{B}_{2}$ at $t_{k}$ the inequality (17) holds in particular for a switching signal $s(t)=1$ for $t \leq t_{k}, s(t)=2$ for $t>t_{k}$. Since for every choice of $v \in \mathbb{R}^{n_{1}}$ there exists a trajectory $\left.w \in \mathfrak{B}_{1}\right|_{(-\infty, 0]}$ s.t. $\left(X_{1}\left(\frac{d}{d t}\right) w\right)\left(0^{-}\right)=v$, using Lemma 3 we conclude that (17) holds, then $\Psi_{12}^{\top}+\Psi_{22} \Pi=0$. Consequently,

$$
\begin{aligned}
\widetilde{\Psi} & =\left[\begin{array}{cc}
\Psi_{11} & -\Pi \Psi_{22} \\
-\Psi_{22} \Pi & \Psi_{22}
\end{array}\right] \\
& =\left[\begin{array}{ll}
\widetilde{\Psi}^{\prime} & 0 \\
0 & 0
\end{array}\right]+\left[\begin{array}{c}
\Pi^{\top} \\
-I_{n_{1}-n_{2}}
\end{array}\right] \Psi_{22}\left[\begin{array}{ll}
\Pi & -I_{n_{1}-n_{2}}
\end{array}\right],
\end{aligned}
$$

where $\widetilde{\Psi}^{\prime}:=\Psi_{11}-\Pi^{\top} \Psi_{22} \Pi$. Pre- and post-multiply (18) by $X_{1}(\zeta)^{\top}$ and $X_{1}(\eta)$ to obtain

$$
\begin{aligned}
& \Psi(\zeta, \eta)=\underbrace{X_{2}(\zeta)^{\top} \tilde{\Psi}^{\prime} X_{2}(\eta)}_{=: \Psi^{\prime}(\zeta, \eta)} \\
& +X_{1}(\zeta)^{\top}\left[\begin{array}{c}
\Pi^{\top} \\
-I_{\left(n_{1}-n_{2}\right)}
\end{array}\right] \Psi_{22}\left[\begin{array}{ll}
\Pi & -I_{\left(n_{1}-n_{2}\right)}
\end{array}\right] X_{1}(\eta) .
\end{aligned}
$$

Since $\Psi_{1}$ is a Lyapunov function for ker $R_{1}\left(\frac{d}{d t}\right)$, there exists $V \in \mathbb{R}^{\mathrm{w} \times \mathrm{w}}[\xi]$ such that $(\zeta+\eta) \Psi_{1}(\zeta, \eta)=$ $-Q(\zeta)^{\top} Q(\eta)+V(\zeta)^{\top} R_{1}(\eta)+R_{1}(\zeta)^{\top} V(\eta)$. We now show that there exists $M \in \mathbb{R}^{\mathrm{w} \times \mathrm{w}}[\xi]$ such that $V=M R_{2}$.
From Prop. 4.3 of [16] it follows that $V(\xi)=$ $\lim _{\mu \rightarrow \infty} \mu R_{1}(\mu)^{-\top} \Psi_{1}(\mu, \xi)$; substituting (19) in this expression we obtain

$$
\begin{aligned}
& V(\xi)=\lim _{\mu \rightarrow \infty}\left(\mu R_{1}(\mu)^{-\top} X_{2}(\mu)^{\top} \tilde{\Psi}^{\prime} X_{2}(\eta)\right. \\
& +\mu R_{1}(\mu)^{-\top} X_{1}(\mu)^{\top}\left[\begin{array}{c}
\Pi^{\top} \\
-I_{\left(n_{1}-n_{2}\right)}
\end{array}\right] \Psi_{22} \\
& \left.\left[\begin{array}{ll}
\Pi & -I_{\left(n_{1}-n_{2}\right)}
\end{array}\right] X_{1}(\eta)\right) .
\end{aligned}
$$

Since $R_{2} R_{1}^{-1}$ is strictly proper, the first term goes to zero. Now $\left[\begin{array}{ll}\Pi & -I_{n_{1}-n_{2}}\end{array}\right] X_{1}(\xi)=-X_{1}^{\prime}(\xi)+\Pi X_{2}(\xi)$ and consequently

$$
\begin{aligned}
& V(\xi)=-\mu R_{1}(\mu)^{-\top} X_{1}^{\prime \top}(\mu) \Psi_{22}\left[\begin{array}{ll}
\Pi & -I_{\left(n_{1}-n_{2}\right)}
\end{array}\right] X_{1}(\xi) \\
& +\lim _{\mu \rightarrow \infty} \underbrace{\mu R_{1}(\mu)^{-\top} X_{2}(\mu)^{\top}}_{\rightarrow 0} \Pi^{\top} \Psi_{22}\left[\begin{array}{ll}
\Pi & -I_{\left(n_{1}-n_{2}\right)}
\end{array}\right] X_{1}(\xi) \\
& =-\left[\begin{array}{ll}
0_{\left(n_{1}-n_{2}\right) \times \mathrm{w}} & K^{\prime \top}
\end{array}\right] \Psi_{22}\left[\begin{array}{ll}
\Pi & -I_{\left(n_{1}-n_{2}\right)}
\end{array}\right] X_{1}(\xi),
\end{aligned}
$$

where $K^{\prime} \in \mathbb{R}^{\mathrm{w} \times \mathrm{w}}$ is a nonsingular matrix, as proved in Lemma 4 and 5. That $V$ has the right factor $R_{2}$ follows from the following argument. Observe that $\left[\begin{array}{ll}\Pi & -I_{\left(n_{1}-n_{2}\right)}\end{array}\right]\left[\begin{array}{l}X_{2}(\xi) \\ X_{1}^{\prime}(\xi)\end{array}\right]=X_{1}^{\prime}(\xi) \bmod R_{2}-X_{1}^{\prime}(\xi)$. Write $X_{1}^{\prime}(\xi) R_{2}(\xi)^{-1}=P(\xi)+S(\xi)$, with $S(\xi)$ a strictly proper polynomial matrix and $P \in \mathbb{R}^{\left(n_{1}-n_{2}\right) \times \mathrm{w}}[\xi]$; then $\Pi X_{2}(\xi)-X_{1}^{\prime}(\xi)=X_{1}^{\prime}(\xi)-P(\xi) R_{2}(\xi)-$ $X_{1}^{\prime}(\xi)=-P(\xi) R_{2}(\xi)$. This proves that $V(\xi)=$ $\left[\begin{array}{ll}0_{\left(n_{1}-n_{2}\right) \times \mathrm{w}} & K^{\prime \top}\end{array}\right] \Psi_{22} P(\xi) R_{2}(\xi)=: M(\xi) R_{2}(\xi)$.

The equality $(\zeta+\eta) \Psi_{1}(\zeta, \eta)=-Q(\zeta)^{\top} Q(\eta)+$ $R_{2}(\zeta)^{\top} M(\zeta)^{\top} R_{1}(\eta)+R_{1}(\zeta)^{\top} M(\eta) R_{2}(\eta)$, together with rank $Q(j \omega)=$ w for all $\omega \in \mathbb{R}$ and $R_{1}$ being Hurwitz, prove strict positive-realness of $M R_{2} R_{1}^{-1}$. That $M R_{2} R_{1}^{-1}$ is strictly proper follows from $Q R_{1}^{-1}$ being strictly proper and Th. 2 . This concludes the proof.

\section{REFERENCES}

[1] F. Blanchini. Nonquadratic Lyapunov functions for robust control. Automatica, 31(3):451-461, 1995.

[2] M. Bonilla and M. Malabre. Description of switched systems by implicit representations. 51st IEEE Decis. Contr. Conf., pages 3209-3214, 2012.

[3] S. Boyd and Q. Yang. Structured and simultaneous Lyapunov functions for system stability problems. Int. J. Contr., 49(6):2215-2240, 1989

[4] G. Costantini, S. Trenn, and F. Vasca. Regularity and passivity for jump rules in linear switched systems. 52nd IEEE CDC, Firenze, 2013.

[5] A.D. Domínguez-García and S. Trenn. Detection of impulsive effects in switched DAEs with applications to power electronics reliability analysis. Proc. of 49th IEEE Decis. Contr. Conf., Atlanta, GA, pages 5662 - 5667, 2010.

[6] A.H.W. Geerts and J.M. Schumacher. Impulsive-smooth behaviour in multimode systems- part I: State-space and polynomial representations. Automatica, 32(5):747-758, 1996. 
[7] A.H.W. Geerts and J.M. Schumacher. Impulsive-smooth behaviour in multimode systems- part II: Minimality and equivalence. Automatica, 32(6):819-832, 1996.

[8] J. P. Hespanha and A. S. Morse. Switching between stabilizing controllers. Automatica, 38(11):1905-1917, 2002.

[9] J.P. Hespanha, D. Liberzon, and A.R. Teel. Lyapunov conditions for input-to-state stability of impulsive systems. Automatica, 44(11):2735 - 2744, 2008.

[10] T. Kailath. Linear Systems. Prentice-Hall Information and System Sciences Series, 1980.

[11] D. Liberzon. Switching in Systems and Control. Birkhauser. Boston, Basel, Berlin, 2003.

[12] D. Liberzon and S. Trenn. On stability of linear switched differential algebraic equations. Proc. 48th IEEE Decis. Contr. \& 28th Chinese Control Conf., Shanghai (China), pages 2156 - 2161, 2009.

[13] D. Liberzon and S. Trenn. Switched nonlinear differential algebraic equations: Solution theory, Lyapunov functions, and stability. Automatica, 48(5):954-963, 2012.

[14] H. Lin and P.J. Antsaklis. Stability and stabilizability of switched linear systems: A survey of recent results. IEEE Trans. Aut. Contr., 54(2):308-322, 2009.

[15] J. Paxman and G. Vinnicombe. Stability of reset switching systems. Proceedings of the European Control Conference, 2003. Cambridge, U.K.

[16] R. Peeters and P. Rapisarda. A two-variable approach to solve the polynomial Lyapunov equation. Syst. Contr. Lett., 42:117126, 2001.

[17] L. Peng and B. Lehman. Performance prediction of dc-dc converters with impedances as loads. IEEE Trans. Pow. Electr., 19(1):201-209, 2004.

[18] J.W. Polderman. Proper elimination of latent variables. Syst. Contr. Lett., 32:261-269, 1997.

[19] J.W. Polderman and J.C. Willems. Introduction to Mathematical System Theory: A Behavioral Approach. Springer, Berlin, 1997.

[20] P. Rapisarda and C. Kojima. Stabilization, Lyapunov functions, and dissipation. Syst. Contr. Lett., 59(12):806-811, 2010.

[21] P. Rapisarda and P. Rocha. Positive realness and Lyapunov functions. Proceedings of the 20th International Symposium on Mathematical Theory of Networks and Systems, 2012. Melbourne, Australia.

[22] P. Rapisarda and J.C. Willems. State maps for linear systems. SIAM J. Control Optim., 35(3):1053-1091, 1997.

[23] P. Rocha, J.C. Willems, P. Rapisarda, and D. Napp. On the stability of switched behavioral systems. 50th IEEE Decis. Contr. Conf. and European Control Conference (CDC-ECC), pages 1534-1538, 2011.

[24] R. Shorten, K.S. Narendra, and O. Mason. A result on common quadratic Lyapunov functions. IEEE Trans. Aut. Contr., 48(1):110-113, 2003.

[25] R.N. Shorten and K.S. Narendra. On common quadratic Lyapunov functions for pairs of stable LTI systems whose system matrices are in companion form. IEEE Trans. Aut. Contr., 48(4):618-621, 2003.

[26] Z. Sun and S.S. Ge. Switched Linear Systems: Control and Design. Springer-Verlag, New York, 2005.

[27] G. Tao and P.A. Ioannou. Necessary and sufficient conditions for strictly positive real matrices. In Circuits, Devices and Systems, IEE Proceedings G. IET., 137(5), 1990.

[28] S. Trenn. Distributional differential algebraic equations. Ph.D. Thesis, Technische Universität Ilmenau, 2009.

[29] S. Trenn. Switched differential algebraic equations. Dynamics and Control of Switched Electronic Systems. Chapter 6 of: Francesco Vasca and Luigi Iannelli (eds.), Springer Verlag, 2012.
[30] S. Trenn. Stability of switched DAEs. Chapter 3 in: J. Daafouz, S. Tarbouriech and M. Sigalotti (eds.), Hybrid Systems with Constraints, 2013.

[31] S. Trenn and J.C Willems. Switched behaviors with impulses - a unifying framework. 51sst IEEE Decis. Contr. Conf., pages 3203-3208, 2012.

[32] M. Trifkovic, M. Sheikhzadeh, K Nigim, and P. Daoutidis. Modeling and control of a renewable hybrid energy system with hydrogen storage. IEEE Trans. Contr. Syst. Tech., 2013 (In print).

[33] A. van der Schaft and P. Rapisarda. State maps from integration by parts. SIAM J. Control Optim., 49(6):2415-2439, 2011.

[34] G.C. Verghese, B.C. Levy, and T. Kailath. A generalized statespace for singular systems. IEEE Trans. Aut. Contr., 26(4):811831, 1981.

[35] J.C. Willems. The behavioral approach to open and interconnected systems. IEEE Control Systems, 27:46-99, 2007.

[36] J.C. Willems and H.L. Trentelman. On quadratic differential forms. SIAM J. Control Optim., 36:1703-1749, 1998.

[37] F. Xiaogang, L. Jinjun, and F.C. Lee. Impedance specifications for stable dc distributed power systems. IEEE Trans. Power Electr., 17(2):157-162, 2002.

[38] H. Ye, A.N. Michel, and L. Hou. Stability theory for hybrid dynamical systems. IEEE Trans. Aut. Contr., 43(4):461-474, 1998.

[39] C.A. Yfoulis and R. Shorten. A numerical technique for the stability analysis of linear switched systems. Int. J. Contr., 77(11):1019-1039, 2004.

[40] Jun Zhao and D.J. Hill. Dissipativity theory for switched systems. IEEE Trans. Aut. Contr., 53(4):941-953, 2008.

Jonathan C. Mayo-Maldonado received the B.S. and M.Eng. degrees in electrical engineering from Instituto Tecnológico de Ciudad Madero, Mexico, in 2008 and 2010 respectively. He is currently working towards a Ph.D. degree in Electrical and Electronic Engineering in the University of Southampton, UK. His research interests include switched systems and behavioural systems theory.

Paolo Rapisarda obtained a Laurea (M.Sc.) degree in Computer Science at the University of Udine, Italy; and a Ph.D. in Mathematics at the University of Groningen, The Netherlands. He is currently a Senior Lecturer at the Communications, Signal Processing and Control Group of the University of Southampton, UK. He is associate editor of "Systems and Control Letters" and of "Multidimensional Systems and Signal Processing". His research interests include behavioural systems theory, switched systems and data-driven control. For further information visit http://www.ecs.soton.ac.uk/ pr3.

Paula Rocha received the Ph.D. degree in mathematics from the University of Groningen, Groningen, The Netherlands, in 1990 and the "Agregação" degree from the Departamento de Matemática, Universidade de Aveiro, Aveiro, Portugal, in 2001. She is currently a Full Professor with the Departamento de Matemática, University of Aveiro. Her research interests include mathematical systems theory, switched systems and control for biomedical applications. For further information visit http://paginas.fe.up.pt/ mprocha. 